\title{
Multiplex Immunohistochemistry Indicates Biomarkers in Colorectal Cancer
}

\section{Wen Zhang}

First People's Hospital of Yunnan https://orcid.org/0000-0002-0825-1440

\section{Zhengji Song}

First People's Hospital of Yunnan

\section{Baoyue Zhang}

First People's Hospital of Yunnan

Jinli Wang

First People's Hospital of Yunnan

\section{Qiang Guo}

First People's Hospital of Yunnan

\section{Zhiwei Sun}

First People's Hospital of Yunnan

Hui Tang ( $\nabla$ htang1122@aliyun.com )

First People's Hospital of Yunnan

\section{Primary research}

Keywords: Multiplex immunohistochemistry (mlHC), Tumor tissue microarray (TMA), Colorectal cancer (CRC), Diagnosis, Prognosis, Biomarkers, Clinicopathological characteristics

Posted Date: November 17th, 2020

DOl: https://doi.org/10.21203/rs.3.rs-105338/v1

License: (c) (i) This work is licensed under a Creative Commons Attribution 4.0 International License. Read Full License

Version of Record: A version of this preprint was published at Neoplasma on August 30th, 2021. See the published version at https://doi.org/10.4149/neo_2021_210312N324. 


\section{Title page}

Title:

\section{Multiplex Immunohistochemistry Indicates Biomarkers in Colorectal Cancer}

\section{Authors and affiliations:}

Wen Zhang ${ }^{1,2}$, Zhengji Song ${ }^{2}$, Baoyue Zhang ${ }^{2}$, Jinli Wang ${ }^{2}$, Qiang $\mathrm{Guo}^{2}$, Zhiwei Sun ${ }^{3, *}$ and Hui Tang ${ }^{2, *}$

${ }^{1}$ Medical Faculty, Kunming University of Science and Technology, Kunming 650504, Yunnan, China.

${ }^{2}$ Yunnan Digestive Endoscopy Clinical Medical Center, the First People's Hospital of Yunnan Province, Kunming 650032, Yunnan, China.

${ }^{3}$ Department of Hepatobiliary Surgery, the First People's Hospital of Yunnan Province, Kunming 650032, Yunnan, China.

\section{- Correspondence:}

Zhiwei Sun, Male; Department of Hepatobiliary Surgery, Director; The First People' s Hospital of Yunnan Province; The Affiliated Hospital of Kunming University of Science and Technology; No. 157 Jinbi Road, Xishan District, Kunming 650032, Yunnan Province, China; E-mail: 2833570686@qq.com;

Hui Tang, Female; Yunnan Digestive Endoscopy Clinical Medical Center; The First People' s Hospital of Yunnan Province; The Affiliated Hospital of Kunming University of Science and Technology; No. 157 Jinbi Road, Xishan District, Kunming 650032, Yunnan Province, China Tel: +86 8716360 2245; Fax: +86 8716360 2245; E-mail: htang1122@aliyun.com. 


\section{Abstract}

Background: Colorectal cancer (CRC) is the third most commonly diagnosed cancer in males and the second in females, whose survival ratio and indicating biomarkers are limited. The rapid development of multiple immunofluorescence gives rise to widespread applications of this newly advanced technology called multiplex immunohistochemistry (mIHC), which makes it possible to detect several fluorescent proteins on the same tumor tissue microarray (TMA) within the same time and spatial organization. Methods: By taking advantage of this mIHC technology, we detected three tumor-associated antigens (TAA) including the human epidermal growth factor receptor 2 (HER2), the cluster of differentiation 133 (CD133), the programmed death ligand-1 (PD-L1) and one immune-associated macrophage marker, the cluster of differentiation 68 (CD68) in cancer tissues versus para-carcinomatous normal tissues derived from a cohort of 84 CRC patients. Results: All the four markers were upregulated in cancer tissue compared with normal tissues. And the expressions of CD133, HER2, PD-L1 and CD68 were correlated with pathological grade, T stage, tumor size, metastasis, respectively. Accordingly, CD133 and PD-L1 could be applied as potential diagnostic biomarkers for CRC at early stage, while the enrichment of HER2 might act as an advanced indicator in aggressive cancer status of CRC; whereas, CD68 could be potentially considered as an advanced diagnostic indicator in CRC patients, as well as a metastatic promoter in CRC-related TME. Conclusions: The differential expression of these four proteins, as well as their clinicopathological correlation indicates that these four proteins could be utilized as diagnostic and prognostic biomarkers in CRC patients.

\section{Keywords}

Multiplex immunohistochemistry (mIHC), Tumor tissue microarray (TMA), Colorectal cancer (CRC), Diagnosis, Prognosis, Biomarkers, Clinicopathological characteristics 


\section{Background}

Colorectal cancer (CRC) is the third most commonly diagnosed cancer in males and the second in females, accounting for approximately $9.7 \%$ of total cancer cases and approximately $8.5 \%$ of cancer deaths worldwide ${ }^{[1]}$. In China, CRC is the fifth leading cause of cancer-related death, with a total of 191,000 deaths in $2015^{[2]}$.

In spite of novel insights into the molecular basis of CRC, current available therapies do not significantly improve the overall survival (OS) of CRC patients. A considerable proportion of CRC patients develop local recurrence and distant metastasis within 5 years after surgical treatment. Although recent advances have been achieved in multidisciplinary management and treatment of CRC, the disease-free survival of CRC still remains poor ${ }^{[3]}$.

Moreover, using disease risk stratification based on tumor size, lymph node or distant metastases (TNM staging) and histological grading is not sufficient for the prognosis of individual CRC patients ${ }^{[4]}$. Besides, even though serological markers (e.g., CEA) currently used for diagnosis are useful to detect disease recurrence after treatment, they are not so reliable as their changes are not always detectable in patients with CRC. Therefore, additional clinicopathological and prognostic biomarkers are urgently needed.

In light of this, we attempted to analyze the expressions of three tumor-associated antigens (TAA) including the human epidermal growth factor receptor 2 (HER2), the cluster of differentiation 133 (CD133), the programmed death ligand-1 (PD-L1) and one immune-associated macrophage marker, the cluster of differentiation 68 (CD68), as well as their potential clinicopathological value in CRC. Specifically, relying on fluorescent multiplex immunohistochemistry (mIHC) technology on tumor tissue microarray (TMA), we explored the expressions of these markers in cancer tissues versus their para-carcinomatous normal tissues derived from a cohort of $84 \mathrm{CRC}$ patients.

\section{Methods}

\section{Patients}

The HColA180Su14 tumor tissue microarray (TMA) (Panovue, Beijing, China) consisted of paired colorectal adenocarcinoma tissues and matched adjacent normal tissues 
derived from 90 CRC patients, of which a cohort 84 cases were taken into final analyses and 6 cases lacking partial information were unenrolled. Patients underwent surgery from Jan. 2009 to Oct. 2009, and the follow-up information was available from Feb. 2009 to Jul. 2015. The study was conducted under the approval of the Institutional Ethics Committee. All procedures were performed in accordance with the relevant guidelines and regulations. The clinicopathological characteristics of 84 patients were summarized in Table 1.

Table 1. Clinicopathological characteristics of a cohort of 84 CRC patients

\begin{tabular}{|c|c|c|}
\hline Clinicopathological Characteristics $(\mathrm{N}=\mathbf{8 4})$ & Number & Proportion (\%) \\
\hline \multicolumn{3}{|l|}{ Gender } \\
\hline Male & 45 & $53.57 \%$ \\
\hline Female & 39 & $46.43 \%$ \\
\hline \multicolumn{3}{|l|}{ Age (years) } \\
\hline$\leqslant 65$ & 44 & $52.38 \%$ \\
\hline$>65$ & 40 & $47.62 \%$ \\
\hline \multicolumn{3}{|l|}{ Tumor Size (cm) } \\
\hline $\mathrm{V} \leqslant 5 \mathrm{~cm}^{3}$ & 11 & $13.10 \%$ \\
\hline$V>5 \mathrm{~cm}^{3}$ & 73 & $86.90 \%$ \\
\hline$L \leqslant 5 \mathrm{~cm}$ & 35 & $41.67 \%$ \\
\hline $\mathrm{L}>5 \mathrm{~cm}$ & 49 & $58.33 \%$ \\
\hline \multicolumn{3}{|l|}{ T Stage } \\
\hline T1 & 1 & $1.19 \%$ \\
\hline $\mathrm{T} 2$ & 10 & $11.90 \%$ \\
\hline T3 & 47 & $55.95 \%$ \\
\hline T4 & 26 & $30.95 \%$ \\
\hline \multicolumn{3}{|l|}{ Lymph Node (N Stage) } \\
\hline Negative (N0) & 57 & $67.86 \%$ \\
\hline Positive (N1a, b-N2a, b) & 27 & $32.14 \%$ \\
\hline Metastasis (M Stage) & & \\
\hline
\end{tabular}




\begin{tabular}{|c|c|c|}
\hline Negative (M0) & 81 & $96.43 \%$ \\
\hline Positive (M1a, b) & 3 & $3.57 \%$ \\
\hline \multicolumn{3}{|l|}{ TNM Stage } \\
\hline 1 & 10 & $11.90 \%$ \\
\hline IIA & 34 & $40.48 \%$ \\
\hline IIB & 10 & $11.90 \%$ \\
\hline IIC & 1 & $1.19 \%$ \\
\hline IIIA & 1 & $1.19 \%$ \\
\hline IIIB & 17 & $20.24 \%$ \\
\hline IIIC & 8 & $9.52 \%$ \\
\hline IVA & 1 & $1.19 \%$ \\
\hline IVB & 2 & $2.38 \%$ \\
\hline \multicolumn{3}{|l|}{ Pathological Grade } \\
\hline I & 5 & $5.95 \%$ \\
\hline II & 65 & $77.38 \%$ \\
\hline III & 13 & $15.48 \%$ \\
\hline IV & 1 & $1.19 \%$ \\
\hline \multicolumn{3}{|l|}{ Histology } \\
\hline Adenocarcinoma & 72 & $85.71 \%$ \\
\hline Canalicular adenoma & 7 & $8.33 \%$ \\
\hline Mucinous adenocarcinoma & 4 & $4.76 \%$ \\
\hline Signet-ring cell carcinoma & 1 & $1.19 \%$ \\
\hline \multicolumn{3}{|l|}{ Differentiation } \\
\hline Well & 19 & $22.62 \%$ \\
\hline Moderate/Poor & 65 & $77.38 \%$ \\
\hline \multicolumn{3}{|l|}{ Disease status at last follow-up } \\
\hline Survival & 51 & $60.71 \%$ \\
\hline Death & 33 & $39.29 \%$ \\
\hline
\end{tabular}

Sample and tissue microarray (TMA) preparation 
TMAs were made based on pathology diagnosis of each tissue. Firstly, Formalin-fixed, paraffin-embedded tumor samples were identified and specimens were reviewed on hematoxylin and eosin stain by an independent surgical pathologist to confirm the presence of tumor and adjacent normal tissue ${ }^{[5]}$.Then the pathologist circled at least two representative tumor areas from each donor block. Next, Core cylinders $(1 \mathrm{~mm})$ were punched from each of these areas and deposited into a recipient paraffin block to form the TMAs. Finally, consecutive 6-mm-thick TMA sections were cut and placed on charged Poly-L-lysine-coated slides for subsequent IHC analyses ${ }^{[6]}$.

\section{Fluorescent mIHC of TMA}

For mIHC staining, the multiplex IHC antibodies for the CD133, HER2, PD-L1 and CD68 were optimized by concentration and application order, meantime, the spectral library was built based on the single-stained slides ${ }^{[7]}$. Then the multiplex immunofluorescence staining and multispectral imaging of the four proteins were obtained on one TMA slide by using PANO 7-plex IHC Kit (cat 0004100100, Panovue, Beijing, China). Briefly, the slide was deparaffinized by xylene $10 \mathrm{~min}$ for three times, followed by $100 \%$ ethanol, $95 \%$ ethanol, $85 \%$ ethanol, and $75 \%$ ethanol for 5 min, respectively.After rinsing in distilled water for 5 min, slide was pretreated with $100 \mathrm{ml}$ antigen retrieval solution (Citric acid solution, pH6.0/pH9.0) by microwaving method (45 seconds on $100 \%$ power, then 15 min on $20 \%$ power) and transferred to 1xTBST containing slide jar to mix well. The slide was then blocked in $10 \%$ blocking solution for $10 \mathrm{~min}$, and followed by staining respectively with primary antibody against HER2, CD133, PD-L1 or CD68 for 1 hour at room temperature. After washing the slide with 1xTBST 3 min twice, the slide was incubated with polymer HRP-anti-mouse/rabbit IgG secondary antibody for $10 \mathrm{~min}$ at RT. Then slide was covered by Tyramide (TSA)-conjugated fluorophore (TSA Fluorescence Kits, Panovue, Beijing, China) at 1:100 dilution and incubated for $10 \mathrm{~min}$ at RT. Finally TSA was vacuumed off, and slide was washed with 1xTBST 3 min twice for next staining procedure. For every additional marker in the multiplex immunofluorescence assay ${ }^{[8]}$, the process was repeated by microwave heat-treating the slide for antigen retrieval, followed directly by one primary antibody staining in each procedure circle ordered as HER2, CD133, PD-L1, CD68, respectively, and then 
downstream procedures as mentioned above. Nuclei were counterstained with 4'-6'-diamidino-2-phenylindole (DAPI, SIGMA-ALDRICH, D9542) after all the human antigens had been labelled. The detailed information for primary antibodies was summarized in Table 2.

Table 2. Primary antibodies used for mIHC staining

\begin{tabular}{cccccc}
\hline Antibodies & Dilution & Antibody Type & Clone\# & Catalogue\# & Vendor \\
\hline CD133 & $1: 1000$ & Rabbit monoclonal & D4W4N & CST86781 & Cell Signaling Technology \\
HER2 & $1: 200$ & Rabbit monoclonal & BP6020 & BX50015 & Biolynx \\
PD-L1 & $1: 500$ & Rabbit monoclonal & BP6141 & BX00005 & Biolynx \\
CD68 & $1: 800$ & Rabbit monoclonal & BP6036 & BX50031 & Biolynx \\
\hline
\end{tabular}

\section{Multispectral imaging}

To obtain multispectral images, the stained slide was scanned using the Ploaris System (PerkinElmer, Waltham, Massachusetts, US), which captures the fluorescent spectra at 20-nm wavelength intervals from 420 to $720 \mathrm{~nm}$ with identical exposure time; the scans were combined to build a single stack image with high contrast and accuracy.

\section{Scoring multispectral images}

InForm Image Analysis Software (Perkin Elmer, Waltham, Massachusetts, US) was used to batch analysis of multispectral images from the experiment ${ }^{[9]}$. Firstly, images of unstained and single-stained sections were used to extract the spectrum of autofluorescence of tissues and each fluorescein, respectively. Then the extracted images were further used to establish a spectral library required for multispectral unmixing by InForm image analysis software. Finally, reconstructed images of sections with the autofluorescence removed were obtained by using this spectral library. For scoring, three to six representative regions of interest for high powered (200X) imaging from every cases were selected. To build algorithm for segment tissues and cells, a few representative multispectral images from the experiment were loaded into InForm software. Then, two Tissue Categories of TUMOR and STROMA were trained according to DAPI signals intensity. Next, the detected tissues compartments were selected and quantified for each stained proteins in slide. Corresponding numbers of total cells and 
positive cells were counted as well. 4 -bin $(0,1+, 2+, 3+)$ scoring system was used to quantify proteins levels by calculating $\mathrm{H}$-score with cell stains. H-score was calculated using the percentages in each bin and ranges from 0 to 300 . Thereby the score results were shown by the percentage positivity of cells with each bin, which included four levels $(0 \sim 1,1 \sim 2,2 \sim 3,3 \sim)$ so as to measuring protein expressions into negative, low, medium and high levels, respectively. To be simplified, H-score with $0 \sim 1$ and $1 \sim 2(0,1+)$ were considered as Low expression, while $2 \sim 3$ and $3 \sim(2+, 3+)$ were as High expression.

\section{Statistics}

The significance of the data from patient specimens was determined by the Mann-Whitney U test. Overall survival (OS) rates were assessed by the Kaplan-Meier test, and the log-rank test was used to plot survival curves. ${ }^{*} P<0.05$ was considered to be significant, ${ }^{* *} P<0.01$ and ${ }^{* * *} P<0.0001$ were considered to be strongly significant. All the analyses were performed with statistics software GraphPad Prism 8.0. 


\section{Results}

\section{Demographics}

For this cohort of 84 CRC patients, a following-up was carried out until 2015 to evaluate a seven-year survival. Among nine clinicopathological characteristics including gender, age, tumor size, T stage, lymph node, metastasis, TNM stage, pathological grade and differentiation, the patients' survival was associated with six characteristics of them. including patients with small tumor size $(\mathrm{L}<5 \mathrm{~cm})$, negative lymph nodes (N0), negative metastasis (M0), early TNM Stage (TNM 1-2), slight Pathological Grade (Grade I-II), and well differentiation have a relative better prognosis than those with large tumor $(L \geqslant 5 \mathrm{~cm})$, positive lymph nodes (N1-2), distant metastasis (M1), late TNM Stage (TNM 3-4), advanced pathological grade (Grade III-IV) and moderate/poor differentiation $(P<0.05$, Table 3, Figure1).

Table 3. Prognostic clinicopathological features of a cohort of 84 CRC patients

\begin{tabular}{lll}
\hline Clinicopathological Features & HR $(95 \mathrm{Cl} \%)$ & $P$ Value \\
\hline Gender (Male vs. Female) & $1.386(0.6997-2.744)$ & 0.3546 \\
Age (years, $\leq 65$ vs. $>65)$ & $1.13(0.5709-2.236)$ & 0.7251 \\
Tumor size (V $\leq 5 \mathrm{~cm}^{3}$ vs. V $\left.>5 \mathrm{~cm}^{3}\right)$ & $0.1746(0.06747-0.4519)$ & 0.0501 \\
\multicolumn{1}{c}{$(\mathrm{L} \leq 5 \mathrm{~cm}$ vs. L>5cm) } & $0.5012(0.2529-0.9933)$ & 0.0609 \\
T stage (T1-2-3 vs. T4) & $0.4644(0.2170-0.9937)$ & $\mathbf{0 . 0 2 3 3}$ \\
N stage (N0 vs. N1-2) & $0.2912(0.1336-0.6345)$ & $\mathbf{0 . 0 0 0 1}$ \\
M stage (M0 vs. M1) & $0.1849(0.01436-2.381)$ & $\mathbf{0 . 0 0 1 5}$ \\
TNM (TNM I-II vs. III-IV) & $0.243(0.1129-0.5230)$ & $<\mathbf{0 . 0 0 0 1}$ \\
Pathological Grade (I-II vs. III-IV) & $0.3381(0.1148-0.9952)$ & $\mathbf{0 . 0 0 3 3}$ \\
Differentiation (W vs. M+P) & $0.2766(0.1277-0.5989)$ & $\mathbf{0 . 0 2 2 2}$ \\
\hline
\end{tabular}



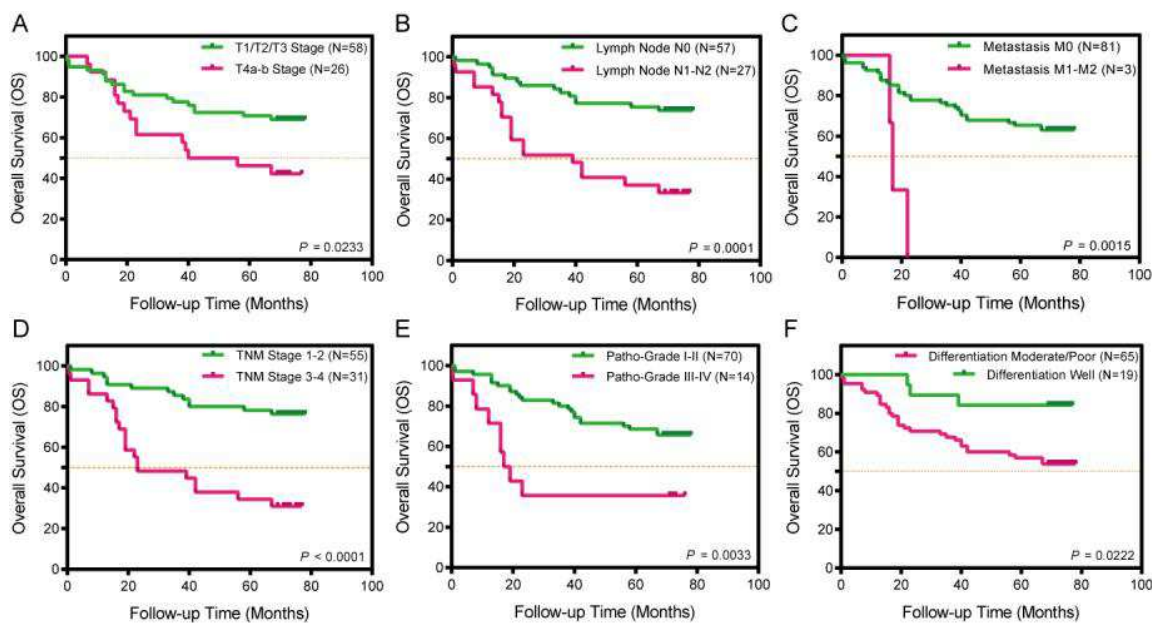

Figure 1. Kaplan-Meier analyzed seven-year overall survival (OS) rates with clinicopathological characteristics. A. T Stage, B. Lymph Node, C. Metastasis, D. TNM Stage, E and F. Pathological Grade as clinical prognostic factors in CRC cancer tissues. Orange line: half of overall survival rates as $50 \%$.

\section{Fluorescent mIHC Profile on TMA Slides of CRC Patients}

To obtain multiple fluorescent images, the TMA slides were firstly trained according to DAPI signals intensity, then detected tissues compartments were selected for each stained protein in slides. After all the four antibodies of HER2, CD133, PD-L1 and CD68 have been performed, the protein expressions were quantified by scoring system to calculate H-score with cell fluorescence. The images of monochromatic proteins in detected tissue compartments and cells were displayed in the upper and middle row ordered as CD133, PD-L1, HER2, and CD68 behind H\&E staining and DAPI image (Figure 2). The multispectral fluorescence of HER2, CD68, PD-L1 and CD133 with DAPI was merged as shown in the bottom big image. The selected images displayed tiuuse from the sigmoid (Figure 2A) and ascending colon (Figure 2B), respectively. 


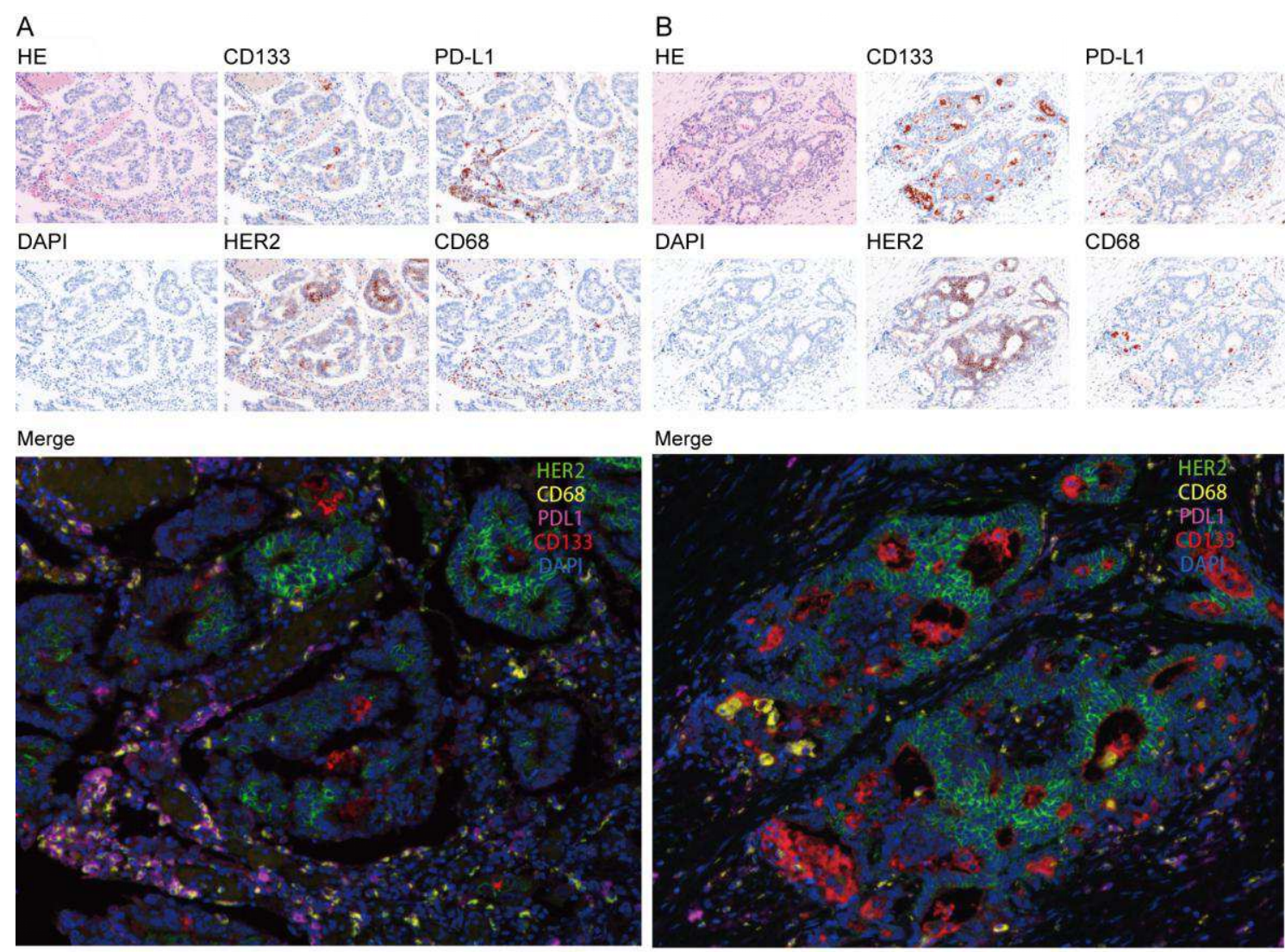

Figure 2. Mono- and pan-chromatic $\mathrm{mIHC}$ profile of $\mathrm{CRC}$ tissue. A-B. Representative images for single and multiple staining in cancer tissues obtained from sigmoideum (A) and ascendens (B) of colon. The upper and middle small images showed the selected tissue compartments stained by H\&E, the raw scanned cell image by DAPI, and the single stained proteins as HER2, CD68, PD-L1 and CD133. The bottom large image showed a merged multispectral fluorescence from HER2, CD68, PD-L1, CD133 and DAPI.

\section{Fluorescent mIHC Determines Significant Markers in CRC Patients}

To explore the potential biomarker in CRC, we compared the expression levels of CD133, PD-L1, HER2 and CD68 between cancer tissue and para-carcinomatous normal tissue of a cohort of 84 CRC patients. No matter monochromatic CD133, PD-L1, HER2 or CD68, or multiple stained combinations, such as bichromatic CD133/PD-L1, CD133/HER2, CD133/CD68, HER2/PD-L1, HER2/CD68 and PD-L1/CD68; and trichromatic CD133/HER2/PD-L1, CD133/PD-L1/CD68, CD133/HER2/CD68 and HER2/PD-L1/CD68; all of them presented higher levels in cancer tissues than in normal tissues (Table 4; Figure 3, 4, 5). As for panchromatic staining of CD133/PD-L1/HER2/CD68, there is no significance between cancer and normal tissues as these four detected proteins were not co-expression in the CRC samples. 
Noticeably, we analyzed all cells in Total firstly, then we divided cells into Tumor cells and Stroma cells, and performed the same analysis again. All the differential expressions of single or multiple stained combinations of these four proteins were analyzed by Mann-Whitney $U$ test and displayed with $P$ value in Table 4. It was shown that the expression trend in Tumor cells was in consistence with that in Total cells, and most of the expressions in Stroma cells were of no differences. Therefore, the comparable expressions of mIHC markers between cancer and normal CRC tissues were only diagrammed from Total cells.

In Total cells, as shown in Figure 3 for monochromatic proteins, Figure 4 for bichromatic combinations, and Figure 5 for trichromatic combinations, except that the expressions of monochromatic CD68, trichromatic CD133/HER2/CD68 and HER2/PD-L1/CD68 were of no differences, other proteins (CD133, PD-L1, HER2; $P<0.05)$ and stained combinations were differentially expressed in cancer tissues compared with adjacent normal tissues $(P<0.01)$.
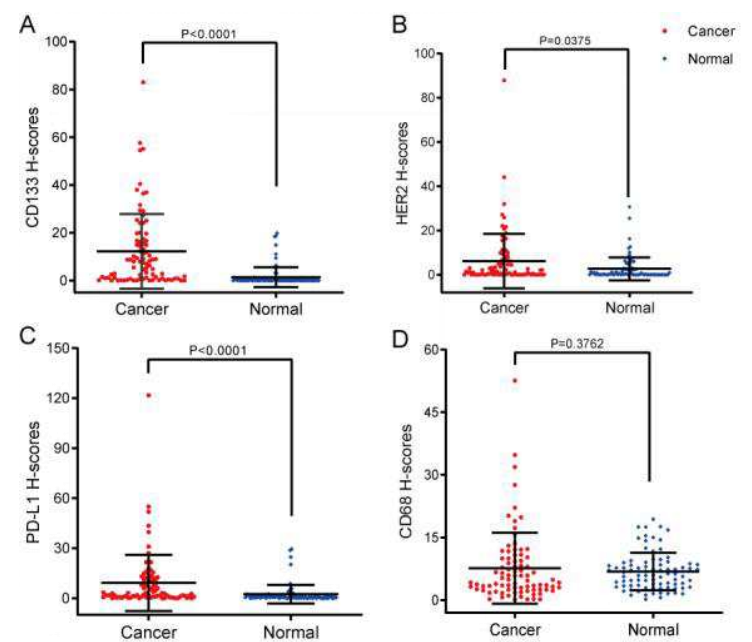

Figure 3. Comparing levels of single stained proteins in Total cells based on H-Scores by mIHC in cancer versus normal tissues in a cohort of $84 \mathrm{CRC}$ patients. Comparing expression of monochromatic marker A. CD133 $(P<0.001)$, B. HER2 $(P=0.0375)$, C. PD-L1 $(P<0.001)$, and D. CD68 (ns. $P=0.3762)$. 

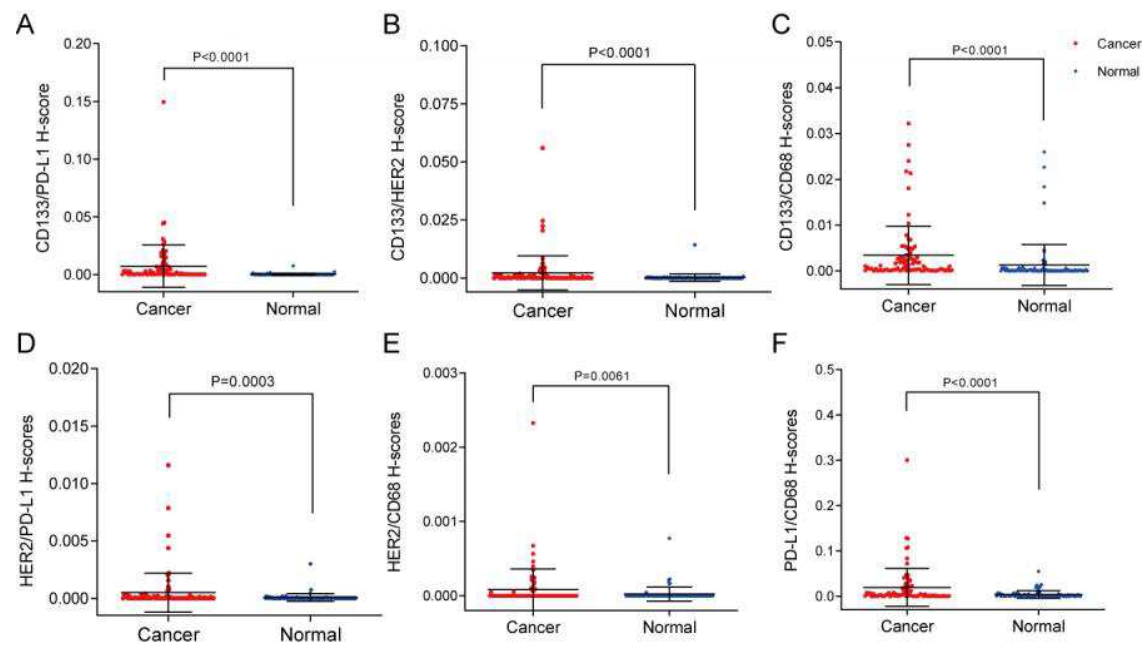

Figure 4. Comparing levels of double stained proteins in Total cells based on H-Scores by $\mathrm{mIHC}$ in cancer versus normal tissues in a cohort of 84 CRC patients. Comparing expression of bichromatic combination A. CD133/PD-L1, B. CD133/HER2, C. CD133/CD68, D. HER2/PD-L1, E. HER2/CD68 and F. PD-L1/CD68. $P<0.01$.
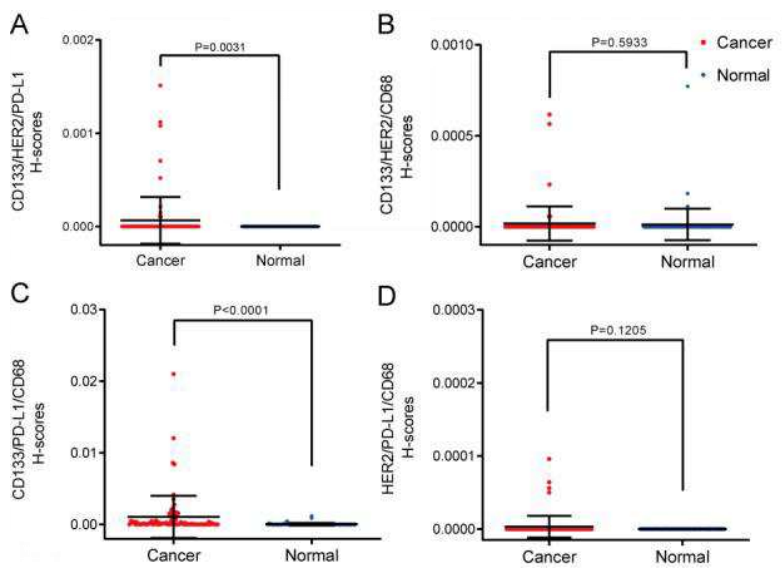

Figure 5. Comparing levels of triple stained proteins in Total cells based on H-Scores by mIHC in cancer versus normal tissues in a cohort of $84 \mathrm{CRC}$ patients. Comparing expression of trichromatic combination A. CD133/HER2/PD-L1 $(P=0.0031), \mathrm{B}$. CD133/PD-L1/CD68 (ns. $P=0.5933)$, C. CD133/HER2/CD68 $(P<0.0001)$ and D. HER2/PD-L1/CD68 (ns. $P=0.1205)$.

Table 4. Differential expressions of mIHC markers in cancer versus normal tissues in a cohort of 84 CRC patients

\begin{tabular}{cccc}
\hline \multirow{2}{*}{ mIHC Markers } & \multicolumn{3}{c}{ Cancer vs. Normal $(\mathbf{N}=\mathbf{8 4})$} \\
\cline { 2 - 4 } & Total cells & Tumor cells & Stroma cells \\
\hline HER2 & $\mathbf{0 . 0 3 7 5}$ & $\mathbf{0 . 0 2 0 7}$ & 0.1064 \\
CD133 & $<\mathbf{0 . 0 0 0 1}$ & $<\mathbf{0 . 0 0 0 1}$ & $<\mathbf{0 . 0 0 0 1}$ \\
\hline
\end{tabular}




\begin{tabular}{cccc}
\hline PD-L1 & $<\mathbf{0 . 0 0 0 1}$ & $<\mathbf{0 . 0 0 0 1}$ & $<\mathbf{0 . 0 0 0 1}$ \\
CD68 & 0.3762 & 0.6407 & 0.2062 \\
CD133/PD-L1 & $<\mathbf{0 . 0 0 0 1}$ & $<\mathbf{0 . 0 0 0 1}$ & $<\mathbf{0 . 0 0 0 1}$ \\
CD133/HER2 & $<\mathbf{0 . 0 0 0 1}$ & $<\mathbf{0 . 0 0 0 1}$ & $\mathbf{0 . 0 1 6 5}$ \\
CD133/CD68 & $<\mathbf{0 . 0 0 0 1}$ & $<\mathbf{0 . 0 0 0 1}$ & $\mathbf{0 . 0 4 8 6}$ \\
HER2/PD-L1 & $\mathbf{0 . 0 0 0 3}$ & $\mathbf{0 . 0 0 0 3}$ & 0.2455 \\
HER2/CD68 & $\mathbf{0 . 0 0 6 1}$ & $\mathbf{0 . 0 0 1 8}$ & $>0.9999$ \\
PD-L1/CD68 & $<\mathbf{0 . 0 0 0 1}$ & $<\mathbf{0 . 0 0 0 1}$ & $<\mathbf{0 . 0 0 0 1}$ \\
CD133/HER2/PD-L1 & $\mathbf{0 . 0 0 3 1}$ & $\mathbf{0 . 0 0 3 1}$ & $>0.9999$ \\
CD133/HER2/CD68 & 0.5933 & 0.2568 & $>0.9999$ \\
CD133/PD-L1/CD68 & $<\mathbf{0 . 0 0 0 1}$ & $<\mathbf{0 . 0 0 0 1}$ & $>0.9999$ \\
HER2/PD-L1/CD68 & 0.1205 & 0.1205 & $>0.9999$ \\
\hline
\end{tabular}

\section{The Correlation between Four Proteins and Clinicopathological Characteristics}

To explore the correlation between four proteins (CD133, PD-L1, HER2, CD68) and nine clinicopathological factors (gender, age, tumor size, T stage, lymph node, metastasis, TNM stage, pathological grade and differentiation, as listed in Table 1), statistic analyses were performed by Mann-Whitney U test. Even though most of the correlations were without significance, all the four markers were found to be involved in one or two specific clinicopathological features among three classfications of cells (Table 5; Figure 6). To be specific, the expression of HER2 was significantly higher in advanced T Stage as T4 $(\mathrm{N}=26)$ than in early T Stages as T1-3 $(\mathrm{N}=58)$ in both Total cells $(P=0.022$, Figure $6 \mathrm{~A})$ and Tumor cells ( $P=0.0263$, Figure 6E). PD-L1 expression was associated with tumor size, but unusually low in tumor with maximum length $<5 \mathrm{~cm}(\mathrm{~N}=49)$ yet high in length $>5 \mathrm{~cm}(\mathrm{~N}=35)$ in Total cells $(P=0.0408$, Figure 6B). As for CD133, its expression was significantly higher in CRC patients at early Pathological Grade I and II $(\mathrm{N}=70)$ than those who were judged as advanced Grade III and IV $(\mathrm{N}=14)$ in both Total cells $(P=0.013$, Figure 6C) and Tumor cells $(P=0.01$, Figure 6F). Notably, the expression of CD68 was also associated with pathological grading in Total cells, but in contrast to CD133, CD68 significantly expressed in Grade III and IV (N=14) than patients as Grade I and II $(\mathrm{N}=70)(P=0.0462$, Figure 6D). Furthermore, CD68 was the 
only one marker with significant expression related to clinical features in Stroma cells, which significantly highly expressed in patients with positive tumor metastasis $(\mathrm{N}=3)$ compared to patients with negative metastasis $(\mathrm{N}=81)(P=0.0327$, Figure 6G). It suggested that $\mathrm{CD} 133$ and PD-L1 probably be the early markers in CRC occurrence, while HER2 and CD68 might make a clue of more aggressive cancer status during CRC progression. 
Table 5. Correlation between potential markers and clinicopathological features in a cohort of 84 CRC patients

\begin{tabular}{|c|c|c|c|c|c|c|c|c|c|c|c|c|}
\hline \multirow{3}{*}{$\begin{array}{c}\text { Clinicopathological features } \\
\text { in cancer tissue } \\
\text { Marker expression }\end{array}$} & \multicolumn{12}{|c|}{$P$ Value of prognostic markers } \\
\hline & \multicolumn{4}{|c|}{ Total cells } & \multicolumn{4}{|c|}{ Tumor cells } & \multicolumn{4}{|c|}{ Stroma cells } \\
\hline & HER2 & CD133 & PD-L1 & CD68 & HER2 & CD133 & PD-L1 & CD68 & HER2 & CD133 & PD-L1 & CD68 \\
\hline Gender (Male vs. Female) & 0.4266 & 0.1347 & 0.5678 & 0.8374 & 0.4585 & 0.1766 & 0.4737 & 0.7959 & 0.5955 & 0.1072 & 0.5978 & 0.9644 \\
\hline Age(years, $\leq 65$ vs. $>65$ ) & 0.8202 & 0.9893 & 0.1389 & 0.3796 & 0.8202 & 0.8342 & 0.1233 & 0.3241 & 0.6765 & 0.7237 & 0.148 & 0.8478 \\
\hline Tumor size $\left(V \leq 5 \mathrm{~cm}^{3}\right.$ vs. $\left.V>5 \mathrm{~cm}^{3}\right)$ & 0.2012 & 0.8548 & 0.4315 & 0.2013 & 0.1701 & 0.9791 & 0.3235 & 0.2061 & 0.8528 & 0.572 & 0.8034 & 0.4463 \\
\hline$(\mathrm{L} \leq 5 \mathrm{~cm}$ vs. $\mathrm{L}>5 \mathrm{~cm})$ & 0.5694 & 0.9568 & 0.0408 * & 0.3113 & 0.5451 & 0.964 & 0.0567 & 0.3907 & 0.5844 & 0.5865 & 0.0584 & 0.8002 \\
\hline T stage (T1-2-3 vs. T4) & 0.022 * & 0.1382 & 0.6002 & 0.6069 & 0.0263 * & 0.1096 & 0.6392 & 0.7256 & 0.1654 & 0.7391 & 0.8135 & 0.9885 \\
\hline N stage (Negative vs. Positive) & 0.0829 & 0.4809 & 0.9091 & 0.5234 & 0.0642 & 0.3551 & 0.6222 & 0.7972 & 0.3501 & 0.389 & 0.567 & 0.9014 \\
\hline M stage (Negative vs. Positive) & 0.7851 & 0.8918 & 0.6646 & 0.2819 & 0.9098 & $>0.9999$ & 0.7093 & 0.1508 & 0.6014 & 0.5038 & 0.8382 & 0.0327 * \\
\hline TNM (TNM 1-2I-II vs. III-IV) & 0.1454 & 0.5618 & 0.7862 & 0.6944 & 0.1058 & 0.41 & 0.9199 & 0.9627 & 0.3322 & 0.3468 & 0.844 & 0.5299 \\
\hline Pathological Grade (I-II vs. III-IV) & 0.4941 & $0.013^{*}$ & 0.5248 & 0.0462 * & 0.4866 & 0.01 * & 0.5547 & 0.0581 & 0.5178 & 0.2193 & 0.3679 & 0.0593 \\
\hline Differentiation (W vs. $\mathrm{H}+\mathrm{L}$ ) & 0.5451 & 0.4902 & 0.6032 & 0.9831 & 0.5451 & 0.5037 & 0.7325 & 0.9072 & 0.9164 & 0.4363 & 0.7824 & 0.8781 \\
\hline
\end{tabular}


*: $P<0.05$. Statistical analysis were performed by Mann-Whitney $U$ test to correlate positive expression of four proteins to related clinical characteristics in Total, Tumor and Stroma cells of cancer tissue, respectively.

A

Total

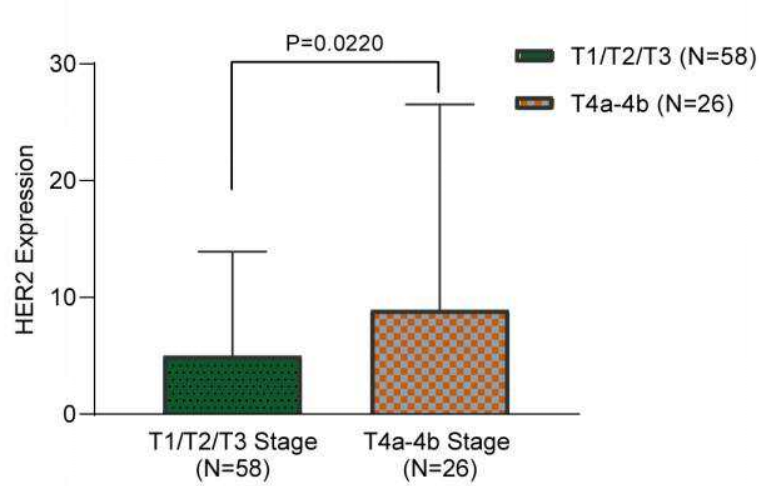

C Pathological Grade

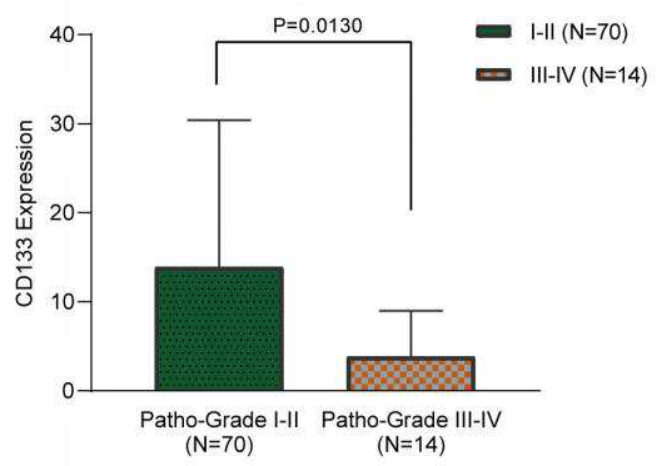

B

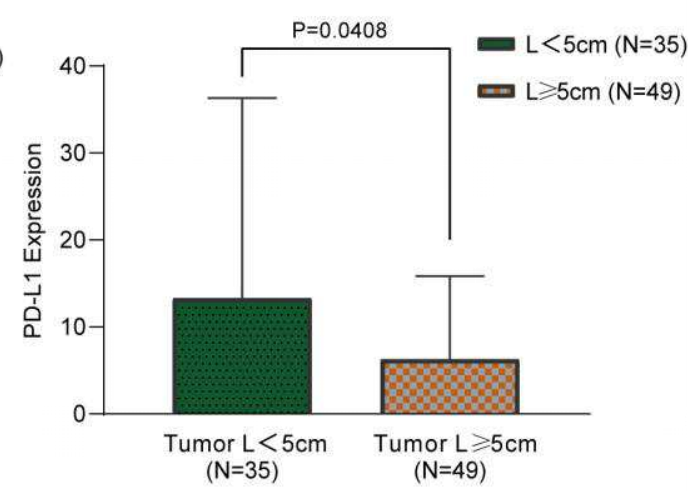

D

Pathological Grade

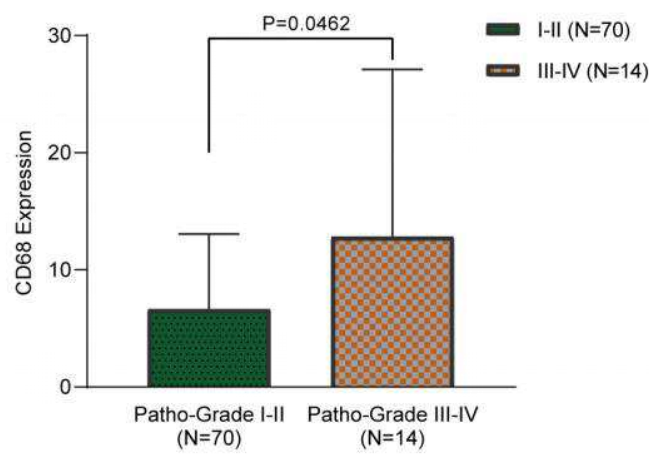

G
E

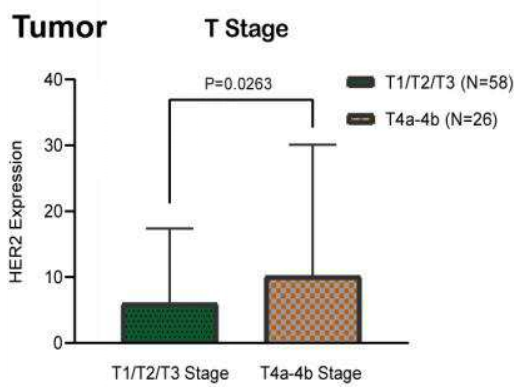

F

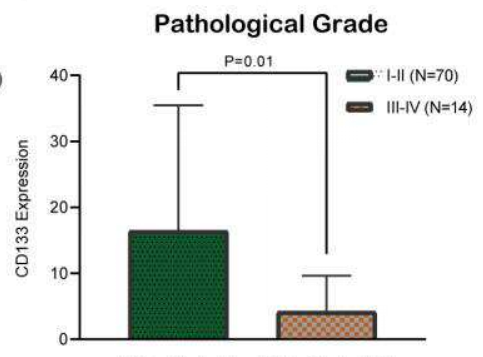

Stroma Metastasis

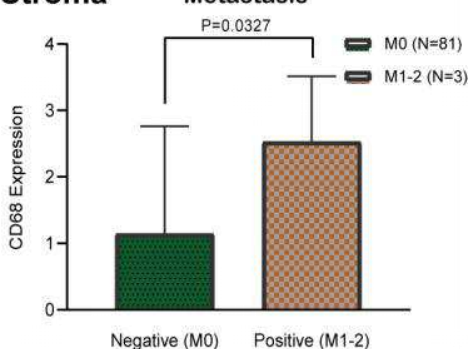

Figure 6. Significant correlations between four markers and clinicopathological characteristics in three cell classifications of CRC cancer tissues. A-D. In Total cells of cancer tissue; E-F. In Tumor cells of cancer tissue; G. In Stroma cells of cancer tissue. A and E. The expression of HER2 was increased in CRC patients at late T4 stage than those at early stages as T1, T2 and T3 . B. The expression of PD-L1 was decreased in small tumor with maximum Length $\geqslant 5 \mathrm{~cm}$ compared in large tumor $\mathrm{L}<5 \mathrm{~cm}$ The levels of $\mathrm{C}$. CD133 in Total similar to F. CD133 in Tumor, were reversely expressed in advanced pathological grading III, IV compared with in Grade I, II to D. CD68 in Total cells. G.CD68 expression was increased in CRC patients with distant metastasis than negative patients. 


\section{Prognostic Markers Associated with Clinicopathological Characteristics in a cohort 84 CRC patients}

To explore the prognostic potential of the four markers in CRC patients, the expression level of each marker was divided into two groups according to the expression level represented by $\mathrm{H}$-score, which was calculated by the fluorescence intensity from three to six regions of each sample. Meanwhile, to better understand the correlation of the expression intensity of each marker with different cell types, the $\mathrm{H}$-score was defined as 0 to $1,1+$ to $2+, 2+$ to $3+$. Thus we classified H-score 0 to $1+$ as Low expression level, while H-score $2+$ to $3+$ as High expression level for each protein, and employed Kaplan-Meier test to analyze the correlation between the four proteins and the seven-year Overall Survial (OS) status of 84 CRC patients.

The univariate analyses of four proteins were performed in Total, Tumor and Stroma cells of CRC Cancer tissues, respectively (Table 6). In both Tumor and Stroma cells, there was no proteins exerting prognostic significance among the $84 \mathrm{CRC}$ patients $(P>0.05$, Table 6$)$. While in Total cells, the only one protein with significant prognostic correlation was CD68 (HR (95 $\mathrm{CI} \%)=4.343(0.4361-43.25) ; P=0.0076$; Figure 7D), compared with insignificant CD133 ( $P=0.3999$, Figure 7A), HER2 $(P=0.6427$, Figure 7B) and PD-L1 $(P=0.9036$, Figure 7C). It demonstrated that CRC patients whose tumors featured High CD68 $(\mathrm{N}=80)$ had a longer OS time than those whose tumors featured Low CD68 (N=4) $(P<0.01$, Figure 7D). The result supported our hypothesis that the elevation of CD68 expression in CRC was partially due to cancer progression of cancer patients, meanwhile partially benefited prognosis of cancer patients at late stage.

Table 6. Association of four proteins with clinical prognosis in a cohort of 84 CRC patients

\begin{tabular}{ccccccc}
\hline \multirow{2}{*}{$\begin{array}{c}\text { Detected } \\
\text { proteins in CRC }\end{array}$} & Total cells & Tumor cells & \multicolumn{2}{c}{ Stroma cells } \\
\cline { 2 - 6 } Cancer (N=84) & HR (95 Cl\%) & P Value & HR (95 Cl\%) & $P$ Value & HR (95 Cl\%) & $P$ Value \\
\hline CD133 & 0.7144 & \multirow{2}{*}{0.3999} & 1.274 & 0.8221 & \\
(Low vs. High) & $(0.2979-1.713)$ & & $(0.5296-3.066)$ & & $(0.4068-1.661)$ &
\end{tabular}




\begin{tabular}{|c|c|c|c|c|c|c|}
\hline HER2 & 0.8436 & \multirow[b]{2}{*}{0.6427} & \multirow{2}{*}{$\begin{array}{c}0.7909 \\
(0.3926-1.593)\end{array}$} & \multirow[b]{2}{*}{0.5218} & 1.751 & \multirow[b]{2}{*}{0.5738} \\
\hline (Low vs. High) & $(0.4165-1.709)$ & & & & $(0.1301-23.58)$ & \\
\hline PD-L1 & 0.9503 & & 1.34 & & 1.007 & \\
\hline (Low vs. High) & $(0.4086-2.210)$ & 0.9036 & $(0.5951-3.016)$ & 0.5129 & (0.5074-1.999) & 0.9839 \\
\hline CD68 & 4.343 & & 1.911 & & 1.172 & \\
\hline (Low vs. High) & $(0.4361-43.25)$ & 0.0076 & $(0.4352-8.391)$ & 0.5138 & $(0.3855-3.560)$ & 0.7649 \\
\hline
\end{tabular}

HR (95 CI\%): Hazard Ratio (95\% Confidence interval)
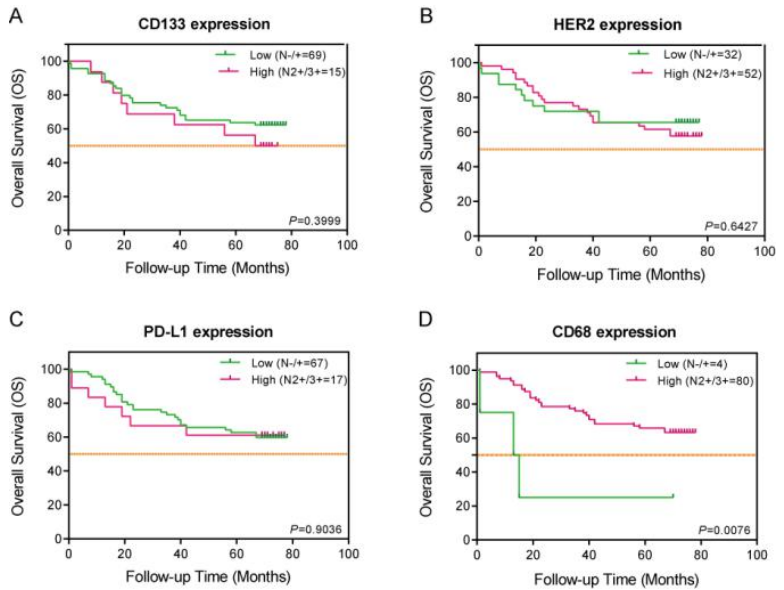

Figure 7. Kaplan-Meier analyzed OS rates with four marker expressions in CRC patients. The High- and Low-expressions of A.

CD133, B. HER2, C. PD-L1 and D. CD68, were associated with seven-year survival status in Total cells of Cancer tissues in 84 CRC patients. Orange dotted line: half of OS rates as 50\%. 


\section{Discussion}

Advances in mIHC techniques and digital pathology platforms allow quantification of multiple proteins at same tissue section and produce continuous data ${ }^{[10]}$. They significantly enrich the data extracted from tumor tissue and facilitate the analysis of relationship between multiple proteins retaining spacial connection ${ }^{[11]}$.

In this study, we performed mIHC on four star molecules to explore the correlation of these well-established biomarkers with CRC. Thereinto, the cluster of differentiation 133 (CD133) is a surface marker of cancer stem cell; the programmed death ligand-1 (PD-L1) is the ligand of the checkpoint molecule PD-1; the human epidermal growth factor receptor 2 (HER2) is a target in breast cancer and related to malignancy and metastasis of tumor; and the cluster of differentiation 68 (CD68) is related to macrophage infiltration in tumor environment (TME). In displayed Total cells of 84 paired cancer-versus-normal tissues from TMA slides of CRC patients, all of these four proteins represented higher expression levels in cancer tissues than in para-carcinomatous normal tissues (Table 4; Figure 3, 4, 5).

Increasing research studies have shown that cancer stem cells (CSCs) with the principal properties of multipotency, infinite proliferation, and self-renewal may be responsible for neoplasm formation, metastasis, recurrence, and therapeutic resistance ${ }^{[12]}$. Recent reports have shown that CSCs are involved in tissue regeneration and carcinogenesis in sporadic CRC, and a variety of molecules have been investigated as putative markers of CSCs in CRC ${ }^{[13]}$. Among them, CD133 (also named AC133 or prominin-1), being a transmembrane glycoprotein mainly expressed in hematopoietic cells, endothelial cells and neuroepithelial cells ${ }^{[14]}$, has been reported as one of the most robust surface marker of CSCs in $\mathrm{CRC}^{[15]}$. CD133 is considered a specific marker of primary colorectal CSCs and the CD133 expression is associated with CRC cell differentiation and tumor size ${ }^{[16]}$.

In 2007, O'Brien ${ }^{[17]}$ found that $\mathrm{CD} 133^{+}$cells in CRC had the ability to initiate tumor growth. Then in 2008, there was a controversial finding reported that both CD133 ${ }^{+}$and CD133metastatic colon cancer cells initiate tumors ${ }^{[18]}$. As for the clinical status of CRC patients, Kashihara $^{[19]}$ demonstrated that high CD133 expression in CRC correlated with poor clinical outcomes. Huang's group ${ }^{[20]}$ confirmed from existing 37 studies-that CD133 would be served as 
a poor predictive indicator in CRC patients, due to CD133 overexpression positively associated with late T category, distant metastasis, lymphatic invasion, vascular invasion, as well as with lower 5-year Overall Survival/Disease-Free Survival (OS/DFS) rate and higher HR of OS/DFS in CRC patients. This finding was almost in line with the study by Wang's team ${ }^{[21]}$. Furthermore, Morteza Akbari ${ }^{[22]}$ confirmed that CD133 has been argued to have prognostic and therapeutic values in CRC along with its related pathways such as Wnt, Notch, and hedgehog. Nevertheless, Hong et al and other studies showed that low expression of CD133 patients were associated with advanced tumor stage and exhibited a poor prognosis ${ }^{[23]}$. And our study was consistent with this report, that the CD133 expression was significantly low in advanced CRC patients with pathological grade III and IV in both Total and Tumor cells of cancer tissue, indicating a poor-prognosis potential of low CD133 in CRC (Figure 6C, 6F).

PD-L1 (also named CD274, B7-H1) interacting with the programmed cell death 1(PDCD1, PD-1) is to form the PD-1/PD-L1 axis, which is an immune checkpoint, and usually up-regulated to create an immunosuppressive tumor microenvironment and help cancer cells escape immune mediated destruction ${ }^{[24]}$. Many studies have confirmed that PD-L1 is mainly expressed on the surface of tumor cells and antigen-presenting cells in various solid malignancies such as squamous cell carcinoma of the head and neck, melanoma, and carcinomas of the brain, thyroid, thymus, esophagus, lung, breast, gastrointestinal tract, colorectum, liver, pancreas, kidney, adrenal cortex, bladder, urothelium, ovary, and skin ${ }^{[25]}$.

In 2013, Shi SJ ${ }^{[26]}$ have demonstrated that PD-L1 may have oncogenic function during colon cancer carcinogenesis. Even though the PD-L1 expression in CRC has not been fully addressed so far, a strong correlation between PD-L1 expression on tumor cells and discrepant clinical outcomes has been observed. Liang et al demonstrated that PD-L1 expression in tumor is an independent predictor of poor CRC prognosis ${ }^{[27]}$. The investigation of Yang ${ }^{[28]}$ also exhibited that the overexpression of PD-L1 indicates a poor prognosis and clinicopathological features. Whereas Masugi ${ }^{[29]}$ found that higher expressions of either PD-1 or PD-L1 correlates with better prognosis of CRC patients. Accordingly, some investigations showed that overexpression of PD-L1 forecasted poor survival in CRC, while others may present negative results ${ }^{[30]}$. In our study, again, PD-L1 expression was low in smaller tumor with, but unusually high in tumor length $>5 \mathrm{~cm}$ (Figure 6B). In addition to that the expressions of both CD133 and 
PD-L1 were obviously significant higher in cancer versus normal tissues in all kinds of detected cells (Total, Tumor and Stroma cells) $(P<0.0001$; Table 4), all these results indicated that CD133 and PD-L1 could serve as diagnostic biomarkers for CRC, and specially be applied as potential biomarker for early screening in CRC patients.

HER2 (also known as HER2/neu, C-erbB2, and p185) is a member of the EGFR family of receptor tyrosine kinases ${ }^{[31]}$. Over the past two decades, HER2 has shown to have an important role in the development and progression of approximately $30 \%$ of breast cancer ${ }^{[32]}$ cases and $10 \%$ of gastric cancer cases ${ }^{[33]}$. Recently, novel studies have indicated that HER 2 is an emerging therapeutic target in $\mathrm{CRC}^{[34]}$. Even the frequency of HER2 overexpression or amplification was low in CRC in Chinese population, the evaluated HER2 status showed clinicopathological association and survival impact on CRC by the HERACLES criteria ${ }^{[35]}$. Our study verified that HER2 expressed significantly, even not so significant as CD133 and PD-L1, in cancer versus normal tissues in both Total and Tumor cells $(P<0.05$; Table 4). But probably because HER2 not only expresses on cell membrane, but also could be secreted into cytoplasm, the differential expression of HER2 was not significant in Stroma cells $(P=0.1064)$. Besides, HER2 displayed a correlation with clinicopathological characteristics lying in that HER2 increased in advanced patients at T4 Stage compared with those at early T1-T3 Stage in both Total and Tumor cells of cancer tissue (Figure 6A, 6E). These results indicated that even the spreading distribution makes HER2 not so specific to be a cancerous biomarker in CRC, its enrichment might act as a clinical indicator in more aggressive cancer status of CRC.

On the contrary, the performance of CD68 is thought-provoking.

As a marker of macrophage, CD68 might exert immunological effect against tumorigenesis. However, in our study, no matter in Total, Tumor or Stroma cells from CRC tissues, the differential expression of CD68 in cancer versus normal tissues was of no significance $(P>0.05$; Table 4), making it failed to become an diagnostic marker for CRC. In contrast, in CRC patients at advanced pathological grading as Grade III or IV, the expression of CD68 was significantly increased when comparing with early Grade I or II (Figure 6D). This could possibly be explained by that as the tumor malignancy gets advanced, there are more inflammatory immune cells, like macrophage, infiltrating within the TME. What's more, as the only one marker with significant expression related to clinical features in stroma cells, CD68 correlated its high 
expression to CRC patients with positive tumor metastasis (Figure 6G). Since stroma cells just indicate the para-cancerous immune infiltration areas, CD68 might exert its tumor-infiltration effect to promote tumor cells to achieve distant metastasis through stroma cells in excellular matrix (ECM). Accordingly, CD68 expression could be accumulated in both advanced cancer and metastatic stroma cells, which brought CD68 to be a potential diagnostic indicator in late period of CRC patients, as well as a metastatic promoter in CRC-related TME.

In terms of survival correlation, it was worth noting that being the only one prognostic marker in our analysis, these CRC patients whose tumors featured high CD68 controversially had a longer survival period than those whose tumors featured low CD68 (Figure 7D). This finding was in line with the result of Zhao et $a \mathrm{a}^{[36]}$, whose study investigated on pan-macrophages, M1-macrophages or M2-macrophages in patients with $\mathrm{CRC}$, and found that unlike other solid tumors, high-density $\mathrm{CD}^{+} 8^{+}$macrophage infiltration can be a good prognostic marker for CRC. On the contrary, the study of Pinto et al ${ }^{[37]}$ indicated that higher CD68 expression in Stage III colorectal tumors is associated with decreased overall survival. Anyhow, since that the sample size in our study for CD68 prognostic analysis was quite small, the result turned out to be not so convincing and needed further confirmation.

These results about CD68 seem to be controversial. However, it is probably attributed to the dual role of macrophage in TME. A variety of studies demonstrated that non-malignant stromal cells, like macrophages, present at the complex TME are active players in cancer progression $^{[38]}$. Macrophages are extremely plastic cells that are able to respond and adapt to external stimuli ${ }^{[39]}$. The most accepted model of macrophage classification currently describes several polarization status between two extreme populations: the M1- like or pro-inflammatory, being the typical activated macrophage to exert immune effect; and the M2-like or anti-inflammatory, mainly consist of tumor associated macrophages (TAMs), which have important roles in the tumor invasive, angiogenic, and metastatic processes ${ }^{[40]}$. Here, we could speculate that in advanced CRC patients, more M1-macrophages were transformed to improve prognosis of CRC patients. Definitely, whether the transformation of macrophage was involved in advanced $\mathrm{CRC}$, and how macrophages display intrinsic plasticity and capacity to adopt intermediate profiles between the two extreme populations, require the use of more specific markers, such as CD80 for M1-macrophage and CD136 for M2-macrophage, as well as a 
combination of strategies to accurately dissect the overall macrophage phenotype in CRC tumors.

\section{Conclusion}

The three tumor-associated antigens (TAA) CD133, PD-L1 and HER2, were significantly increased in all kinds of detected cells in cancer tissue versus adjacent normal tissue in a cohort of 84 CRC patients, while the immune-associated macrophage marker CD68 with no significance. Among these four markers, each was correlated with one specific clinicopathological characteristics to predictively indicate certain tumor status. Potentially, CD133 and PD-L1 could be applied as early, while HER2 and CD68 as late diagnostic biomarkers for CRC patients. In addition, the elevation of CD68 expression in CRC was controversially related with better prognosis, probably due to the polarization of activated M1-like macrophage to exert immune effect. Hence, our Multiplex Immunohistochemistry study effectively provides four important proteins (CD133, PD-L1, HER2 and CD68) as biomarkers for diagnosis and prognosis of colorectal cancer. Since that there are some limitations for this study, for instance, the analytical deviation might be caused by heterogeneity of tumor, specifity of antibodies and small size of sample, subsequently abundant functional assays performed for confirmation of these tumor-related mIHC proteins are of great needs. Moreover, in view of that most of the significant results were obtained from the Total cells of CRC cancer tissue, the importance of TME should be taken into full consideration in cancer research in further studies. Only correlating tumor-associated antigens with occurrence and progression of tumor within the tumor microenvironment could faithfully reflect the tumor status of cancer patients, and thus provide effective diagnostic and therapeutic treatment for them.

\section{List of abbreviations}

CRC: Colorectal cancer

mIHC: multiplex immunohistochemistry

TMA: tumor tissue microarray

TAA: tumor-associated antigens

TME: tumor microenvironment
HER2: human epidermal growth factor receptor 2

CD133: cluster of differentiation 133

PD-L1: programmed death ligand-1

CD68: cluster of differentiation 68 


\section{Declarations}

\section{Ethics approval and consent to participate}

The CRC patients for tumor tissue microarray (TMA) (HColA180Su14; Xinchao, Shanghai, China) were Informed Consent. The study was conducted under the approval of the Institutional Ethics Committee. All procedures were performed in accordance with the relevant guidelines and regulations.

\section{Consent for publication}

As the authors approved the publication of this manuscript.

\section{Availability of data and materials}

The datasets used and/or analysed during the current study are available from the corresponding author on reasonable request.

\section{Competing interests}

The authors declare that they have no competing interests.

\section{Funding}

The research was supported by different funds of the first author and the corresponding author without conflicts. The funds were applied for researching and publishing as following:

National Natural Science Foundation of China [Grants No. 81460463]; Joint Yunnan Provincial Foundation of Science and Technology Department \& Kunming Medical University [2019FE001(-173)]; Foundation of Kunming Key Laboratory of Tumor Molecular and Immune Prevention [2018-1-A-17334]; and Yunnan Digestive Endoscopy Clinical Medical Center Foundation for Health Commission of Yunnan Province [2020LCZXKF-XH06] of [2X2019-01-02] .

\section{Authors' contributions}

All the authors work in the First People's Hospital of Yunnan Province from different departments. For the contributions to this work, TH, SZJ, GQ and ZW partially sponsored this study. Besides, TH was in charge of the design of the research topics; SZW instructed selection of tissue chip; ZW was in charge of data analyses and manuscript writing; ZBY was mainly in charge of connecting with companies, WJL purchased TMA and antibodies. All of the authors engaged much in the detecting and writing work of this study.

\section{Acknowledgments}

All the authors work in the First People's Hospital of Yunnan Province from different departments but engaged much in the detecting and writing work of this study. All the funds and effort are to be appreciated. 


\section{Reference}

[1] BRAY F, FERLAY J, SOERJOMATARAM I, et al. Global cancer statistics 2018: GLOBOCAN estimates of incidence and mortality worldwide for 36 cancers in 185 countries [J]. CA Cancer J Clin, 2018, 68(6): 394-424.

[2] CHEN W, ZHENG R, BAADE P, et al. Cancer statistics in China, 2015 [J]. CA Cancer J Clin, 2016, 66(2): 115-32.

[3] ÖZGüL ÖZDEMIR R, ÖZDEMIR A, OLTULU F, et al. A comparison of cancer stem cell markers and nonclassical major histocompatibility complex antigens in colorectal tumor and noncancerous tissues [J]. Ann Diagn Pathol, 2016, (25)60-3.

[4] ALLEMANI C, WEIR H, CARREIRA H, et al. Global surveillance of cancer survival 1995-2009: analysis of individual data for 25,676,887 patients from 279 population-based registries in 67 countries (CONCORD-2) [J]. Lancet, 2015, 385(9972): 977-1010.

[5] RING K, YEMELYANOVA A, SOLIMAN P, et al. Potential immunotherapy targets in recurrent cervical cancer [J]. Gynecol Oncol, 2017, 145(3): 462-8.

[6] FANG M, LI Y, HUANG K, et al. IL33 Promotes Colon Cancer Cell Stemness via JNK Activation and Macrophage Recruitment [J]. Cancer Res (Tumor and Stem Cell Biology), 2017, 77(10): 2735-45.

[7] YING L, YAN F, MENG Q, et al. PD-L1 expression is a prognostic factor in subgroups of gastric cancer patients stratified according to their levels of CD8 and FOXP3 immune markers [J]. Onco Immunology, 2018, 7(6): e1433520.

[8] MCGUIRE H, SHKLOVSKAYA E, EDWARDS J, et al. Anti-PD-1-induced high-grade hepatitis associated with corticosteroid-resistant $\mathrm{T}$ cells: a case report [J]. Cancer Immunology, Immunotherapy, 2018, 67(4): 563-73.

[9] YANG L, LIU Z, TAN J, et al. Multispectral imaging reveals hyper active TGF- $\beta$ signaling in colorectal cancer [J]. Cancer Biology \& Therapy, 2018, 19(2): 105-12.

[10] FENG Z, PURI S, MOUDGIL T, et al. Multispectral imaging of formalin-fixed tissue predicts ability to generate tumor-infiltrating lymphocytes from melanoma [J]. J Immunother Cancer, 2015, 3(47. [11] HERBST R, SORIA J, KOWANETZ M, et al. Predictive correlates of response to the anti-PD-L1 antibody MPDL3280A in cancer patients [J]. Nature, 2014, 515(7528): 563-7.

[12] VERMEULEN L, DE SOUSA E MELO F, RICHEL D, et al. The developing cancer stem-cell model: clinical challenges and opportunities [J]. Lancet Oncol, 2012, 13(2): e83-9.

[13] ZHOU Y, XIA L, WANG H, et al. Cancer stem cells in progression of colorectal cancer [J]. Oncotarget, 2018, 9(70): 33403-15.

[14] LI Y, XIAO B, TU S, et al. Cultivation and identification of colon cancer stem cell-derived spheres from the Colo205 cell line [J]. Braz J Med Biol Res, 2012, 45(3): 197-204.

[15] RICCI-VITIANI L, LOMBARDI D, PILOZZI E, et al. Identification and expansion of human colon-cancer-initiating cells [J]. Nature, 2007, 445(7123): 111-5.

[16] KAZAMA S, KISHIKAWA J, KIYOMATSU T, et al. Expression of the stem cell marker CD133 is related to tumor development in colorectal carcinogenesis [J]. Asian J Surg, 2018, 41(3): 274-8. 
[17] O'BRIEN C, POLLETT A, GALLINGER S, et al. A human colon cancer cell capable of initiating tumour growth in immunodeficient mice [J]. Nature, 2007, 445(7123): 106-10.

[18] LABARGE M, BISSELL M J T J O C I. Is CD133 a marker of metastatic colon cancer stem cells? [J]. J Clin Invest, 2008, 118(6): 2021-4.

[19] KASHIHARA H, SHIMADA M, KURITA N, et al. CD133 expression is correlated with poor prognosis in colorectal cancer [J]. Hepatogastroenterology, 2014, 61(134): 1563-7.

[20] HUANG R, MO D, WU J, et al. CD133 expression correlates with clinicopathologic features and poor prognosis of colorectal cancer patients: An updated meta-analysis of 37 studies [J]. Medicine (Baltimore), 2018, 97(23): e10446.

[21] WANG B, LI Z, ZHANG F, et al. Clinical significance of stem cell marker CD133 expression in colorectal cancer [J]. Histol Histopathol, 2016, 31(3): 299-306.

[22] AKBARI M, SHOMALI N, FARAJI A, et al. CD133: An emerging prognostic factor and therapeutic target in colorectal cancer [J]. Cell Biol Int, 2020, 44(2): 368-80.

[23] HONG I, HONG S, CHANG Y, et al. Expression of the Cancer Stem Cell Markers CD44 and CD133 in Colorectal Cancer: An Immunohistochemical Staining Analysis [J]. Ann Coloproctol, 2015, 31(3): 84-91.

[24] FUSI A, FESTINO L, BOTTI G, et al. PD-L1 expression as a potential predictive biomarker [J]. Lancet Oncol, 2015, 16(13): 1285-7.

[25] WANG X, TENG F, KONG L, et al. PD-L1 expression in human cancers and its association with clinical outcomes [J]. Onco Targets Ther, 2016, 9(5023-39.

[26] SHI S, WANG L, WANG G, et al. B7-H1 expression is associated with poor prognosis in colorectal carcinoma and regulates the proliferation and invasion of HCT116 colorectal cancer cells [J]. PLoS One, 2013, 8(10): e76012.

[27] LIANG M, LI J, WANG D, et al. T-cell infiltration and expressions of T lymphocyte co-inhibitory B7-H1 and B7-H4 molecules among colorectal cancer patients in northeast China's Heilongjiang province [J]. Tumour Biol, 2014, 35(1): 55-60.

[28] YANG L, XUE R, PAN C J O, et al. Prognostic and clinicopathological value of PD-L1 in colorectal cancer: a systematic review and meta-analysis [J]. Onco Targets Ther, 2019, 12(3671-82.

[29] MASUGI Y, NISHIHARA R, YANG J, et al. Tumour CD274 (PD-L1) expression and T cells in colorectal cancer [J]. Gut, 2017, 66(8): 1463-73.

[30] LI Y, LIANG L, DAI W, et al. Prognostic impact of programed cell death-1 (PD-1) and PD-ligand 1 (PD-L1) expression in cancer cells and tumor infiltrating lymphocytes in colorectal cancer [J]. Mol Cancer, 2016, 15(1): 55.

[31] KUNTE S, ABRAHAM J, MONTERO A J C. Novel HER2-targeted therapies for HER2-positive metastatic breast cancer [J]. Cancer, 2020,

[32] SLAMON D, CLARK G, WONG S, et al. Human breast cancer: correlation of relapse and survival with amplification of the HER-2/neu oncogene [J]. Science, 1987, 235(4785): 177-82.

[33] TERASHIMA M, KITADA K, OCHIAI A, et al. Impact of expression of human epidermal growth factor receptors EGFR and ERBB2 on survival in stage II/III gastric cancer [J]. Clin Cancer Res, 2012, 18(21): 5992-6000. 
[34] LIU F, REN C, JIN Y, et al. Assessment of two different HER2 scoring systems and clinical relevance for colorectal cancer [J]. Virchows Arch, 2020, 476(3): 391-8.

[35] YEH Y, LEE C, CHEN S, et al. HER2 Comprehensive assessment of alteration in a colorectal cancer cohort: from next-generation sequencing to clinical significance [J]. Cancer Manag Res, 2019, 11(7867-75.

[36] ZHAO Y, GE X, XU X, et al. Prognostic value and clinicopathological roles of phenotypes of tumour-associated macrophages in colorectal cancer [J]. J Cancer Res Clin Oncol, 2019, 145(12): 3005-19.

[37] PINTO M, RIOS E, DURãES C, et al. The Two Faces of Tumor-Associated Macrophages and Their Clinical Significance in Colorectal Cancer [J]. Front Immunol, 2019, 10(1875.

[38] HANAHAN D, WEINBERG R J C. Hallmarks of cancer: the next generation [J]. Cell, 2011, 144(5): 646-74.

[39] MURRAY P, ALLEN J, BISWAS S, et al. Macrophage activation and polarization: nomenclature and experimental guidelines [J]. Immunity, 2014, 41(1): 14-20.

[40] CONDEELIS J, POLLARD J J C. Macrophages: obligate partners for tumor cell migration, invasion, and metastasis [J]. Cell, 2006, 124(2): 263-6. 

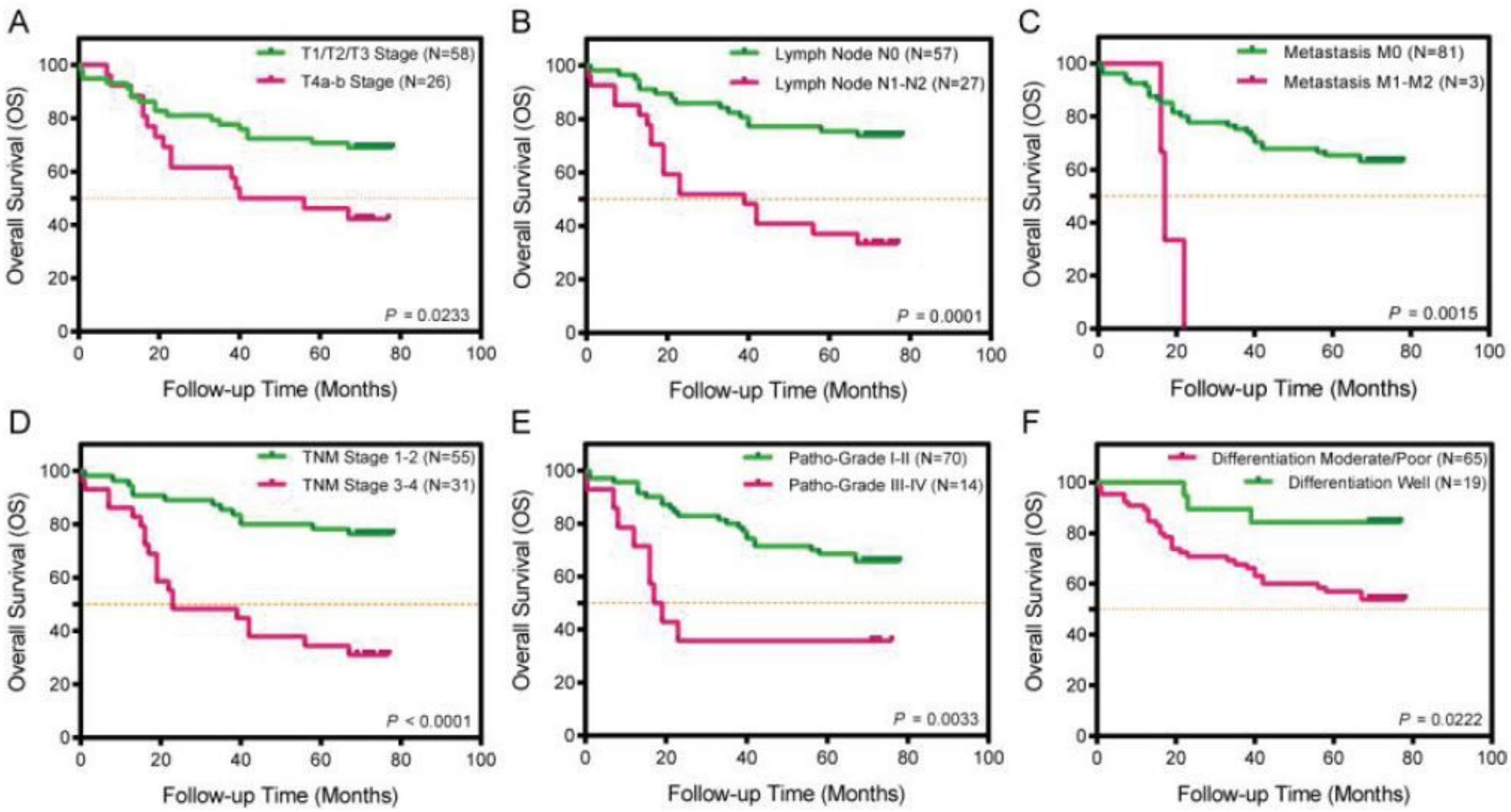

\section{Figure 1}

Kaplan-Meier analyzed seven-year overall survival (OS) rates with clinicopathological characteristics. A. T Stage, B. Lymph Node, C. Metastasis, D. TNM Stage, E and F. Pathological Grade as clinical prognostic factors in CRC cancer tissues. Orange line: half of overall survival rates as $50 \%$. 

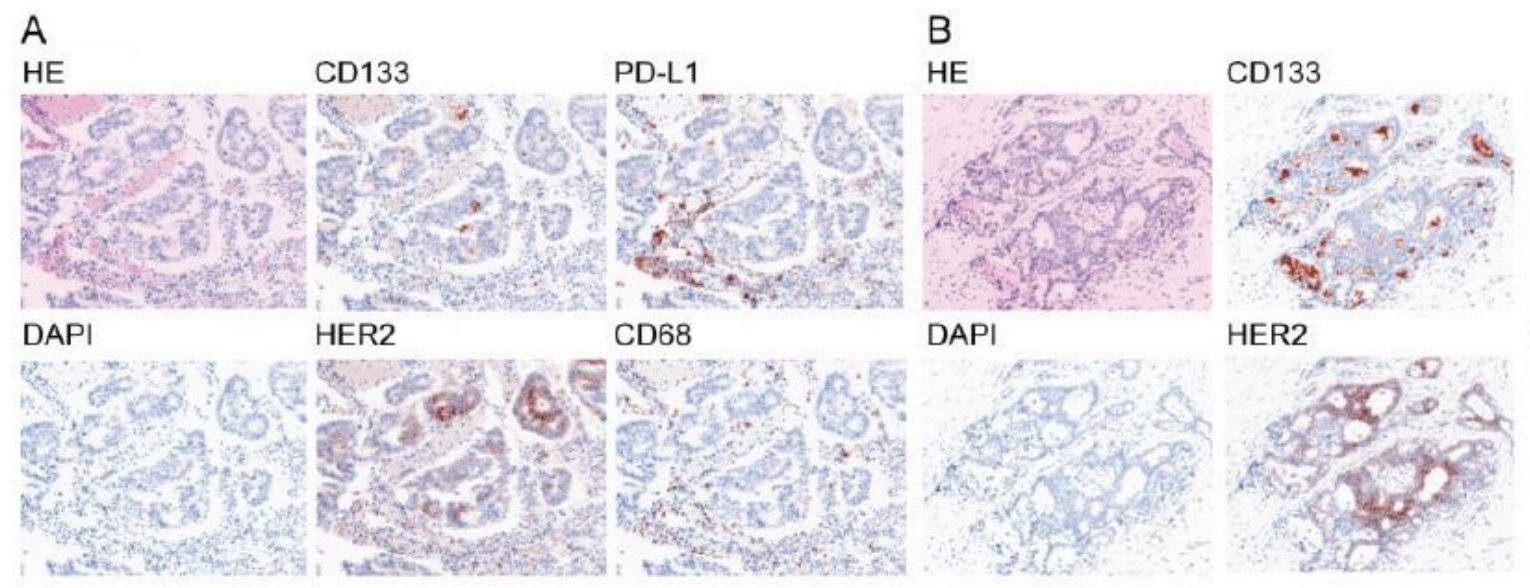

PD-L1

Merge

HER2

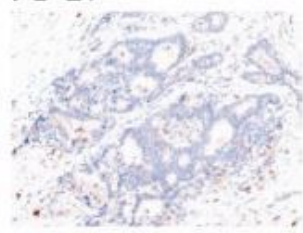

CD68
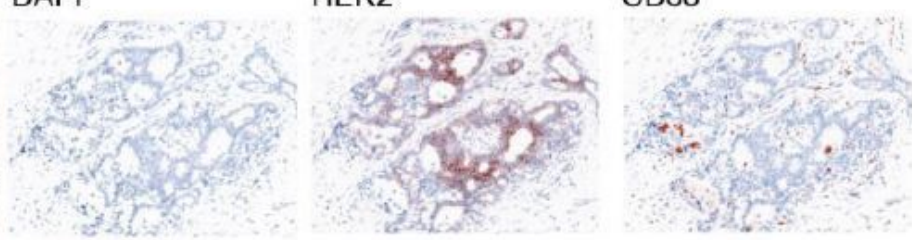

Merge
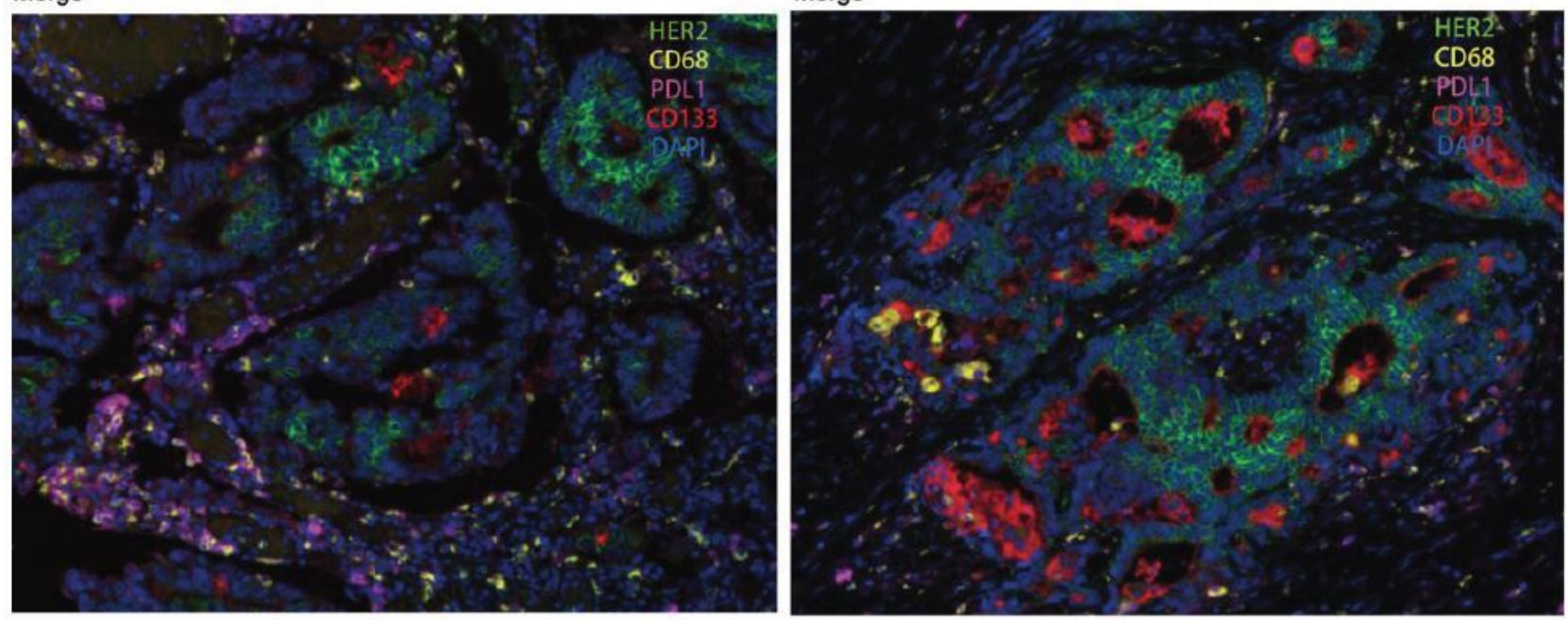

\section{Figure 2}

Mono- and pan-chromatic mIHC profile of CRC tissue. A-B. Representative images for single and multiple staining in cancer tissues obtained from sigmoideum (A) and ascendens $(B)$ of colon. The upper and middle small images showed the selected tissue compartments stained by H\&E, the raw scanned cell image by DAPI, and the single stained proteins as HER2, CD68, PD-L1 and CD133. The bottom large image showed a merged multispectral fluorescence from HER2, CD68, PD-L1, CD133 and DAPI. 

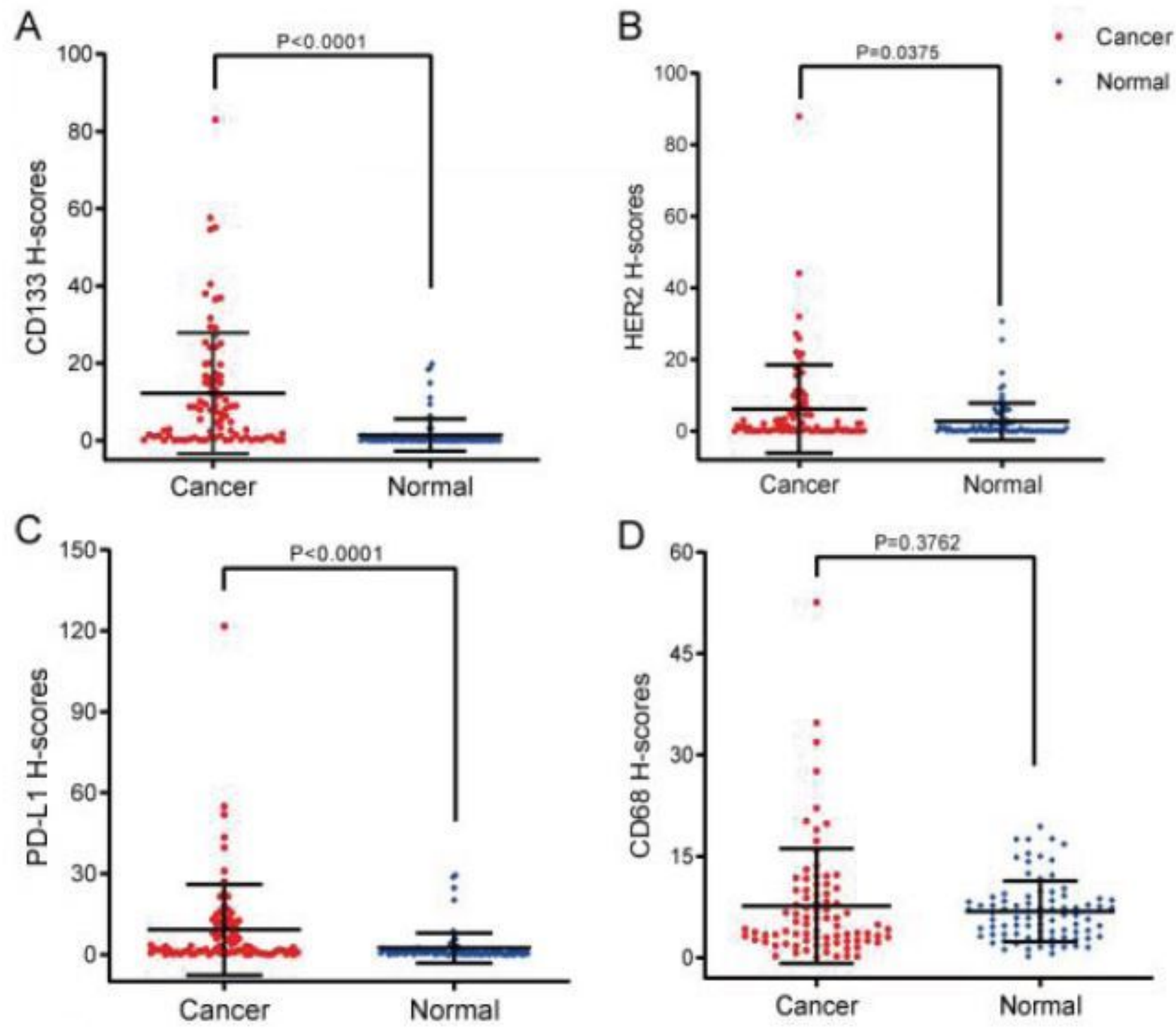

Figure 3

Comparing levels of single stained proteins in Total cells based on $\mathrm{H}$-Scores by $\mathrm{mIHC}$ in cancer versus normal tissues in a cohort of $84 \mathrm{CRC}$ patients. Comparing expression of monochromatic marker $\mathrm{A}$. CD133 ( $P<0.001)$, B. HER2 ( $P=0.0375)$, C. PD-L1 $(P<0.001)$, and D. CD68 (ns. $P=0.3762)$. 

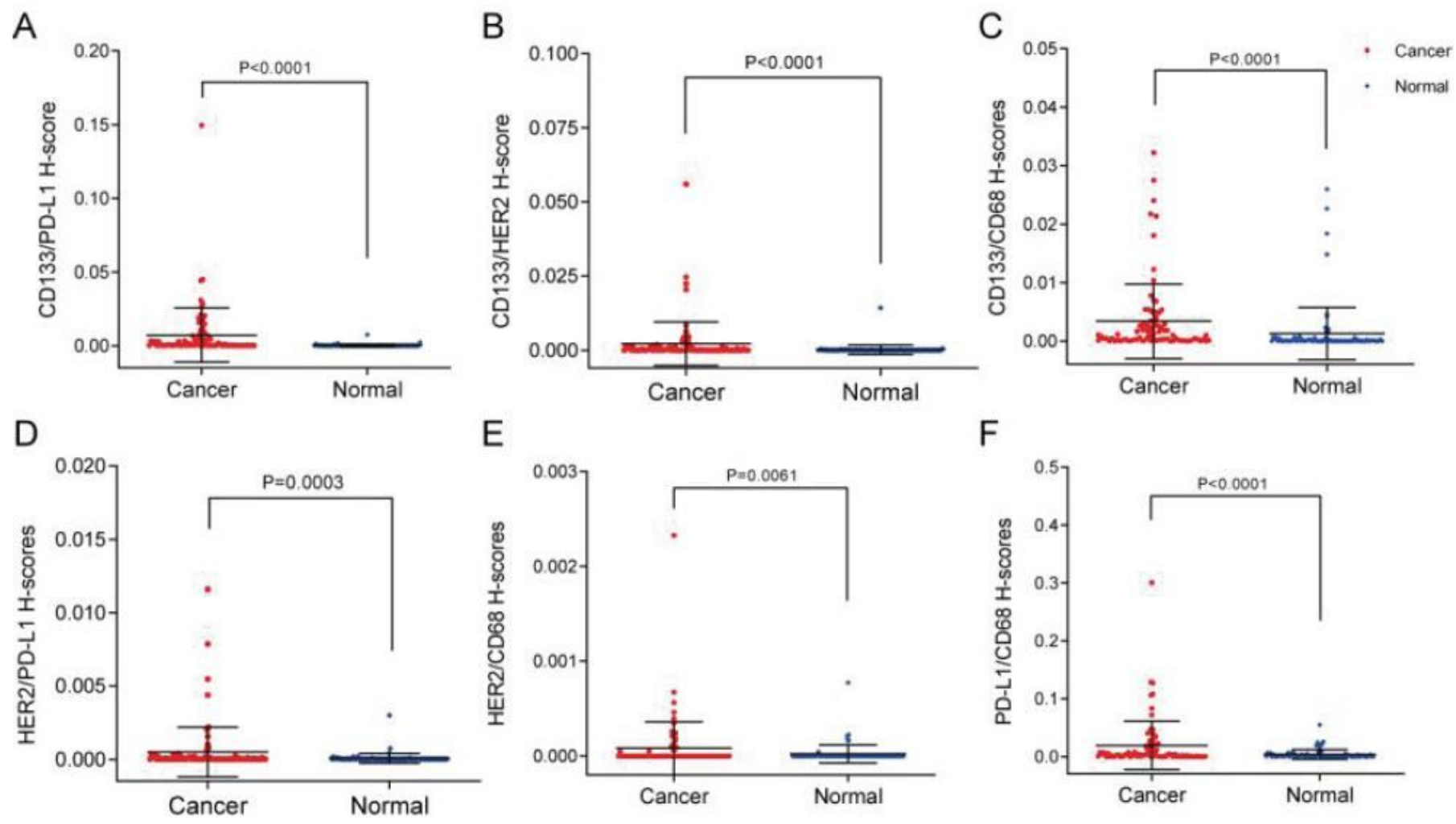

\section{Figure 4}

Comparing levels of double stained proteins in Total cells based on $\mathrm{H}$-Scores by $\mathrm{mIHC}$ in cancer versus normal tissues in a cohort of $84 \mathrm{CRC}$ patients. Comparing expression of bichromatic combination $\mathrm{A}$. CD133/PD-L1, B. CD133/HER2, C. CD133/CD68, D. HER2/PD-L1, E. HER2/CD68 and F. PD-L1/CD68. P< 0.01 . 

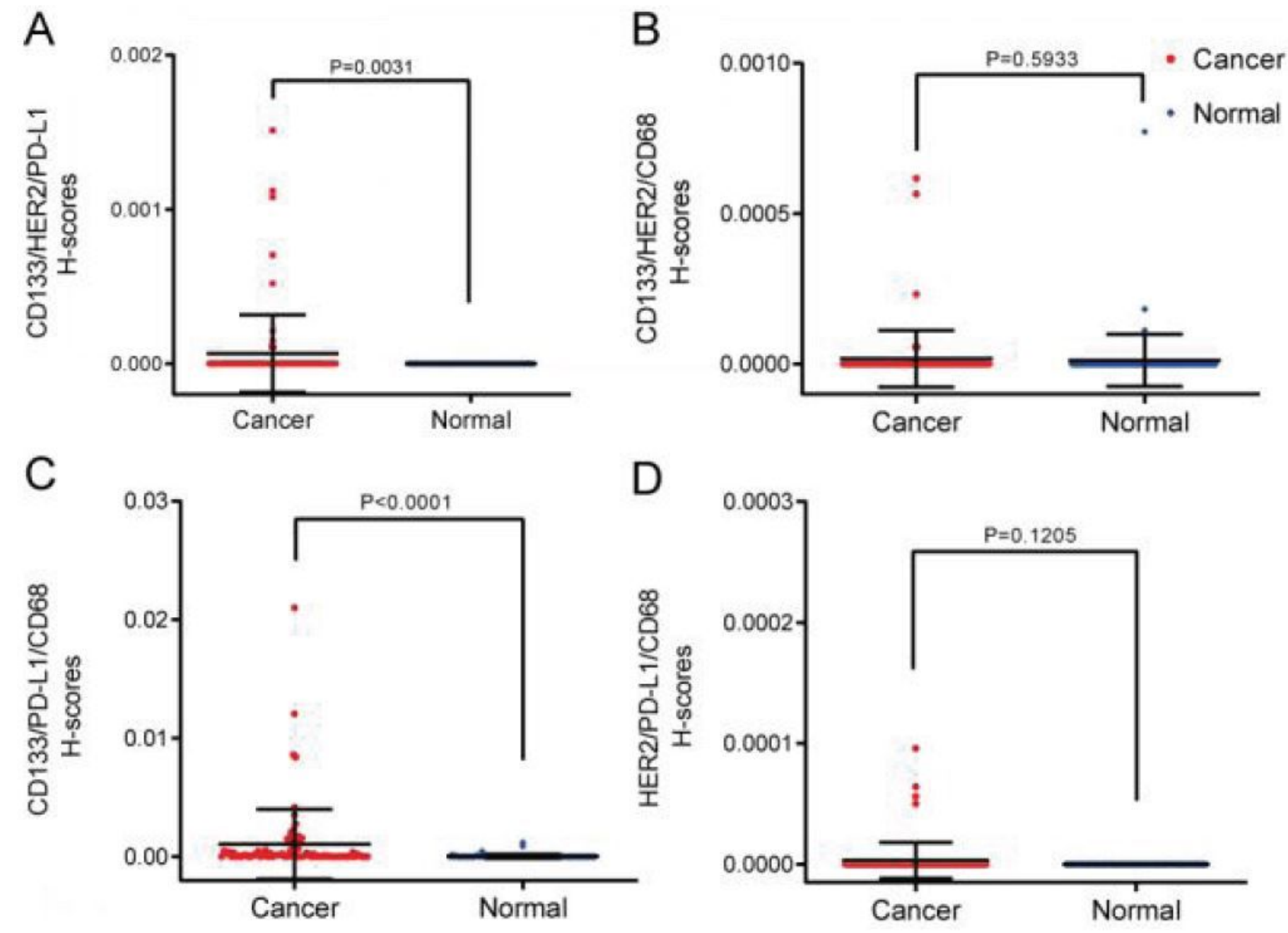

Figure 5

Comparing levels of triple stained proteins in Total cells based on $\mathrm{H}$-Scores by $\mathrm{mIHC}$ in cancer versus normal tissues in a cohort of $84 \mathrm{CRC}$ patients. Comparing expression of trichromatic combination $\mathrm{A}$. CD133/HER2/PD-L1 (P =0.0031), B. CD133/PD-L1/CD68 (ns. P = 0.5933), C. CD133/HER2/CD68 ( $<$ $0.0001)$ and D. HER2/PD-L1/CD68 (ns. $P=0.1205$ ). 
A

Total

T Stage

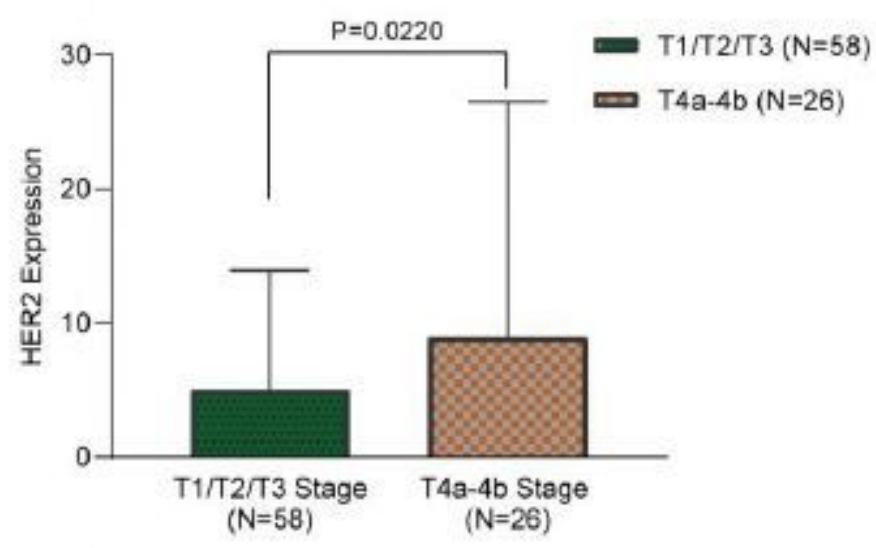

C

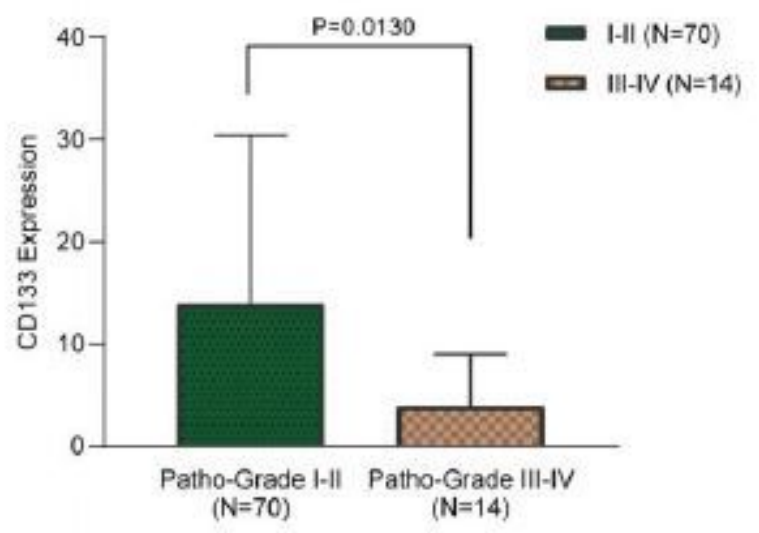

B

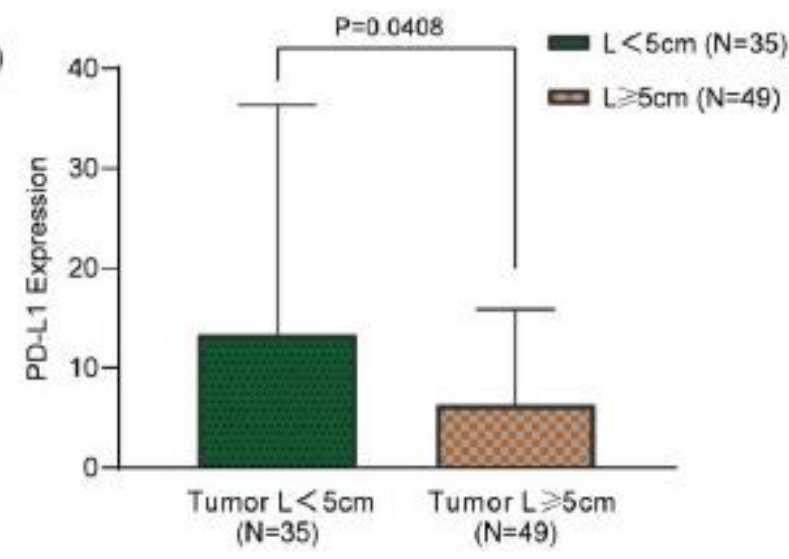

Pathological Grade

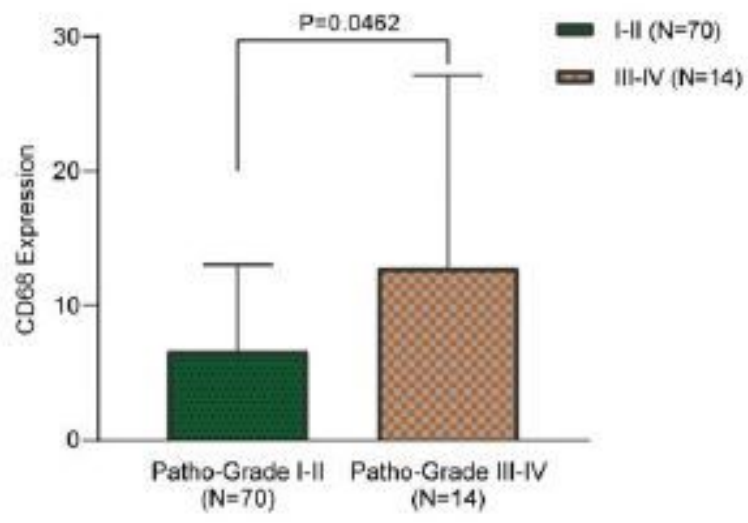

E

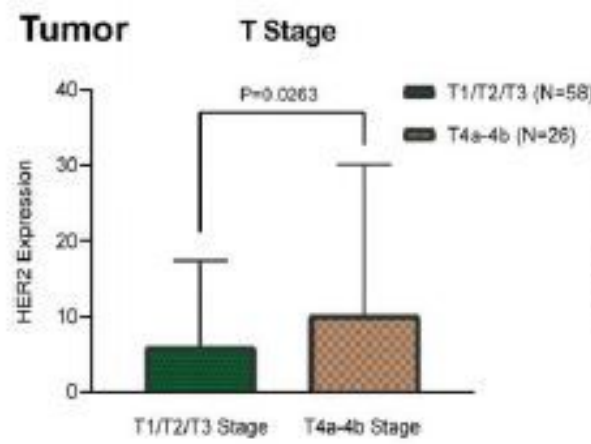

$\mathrm{F}$

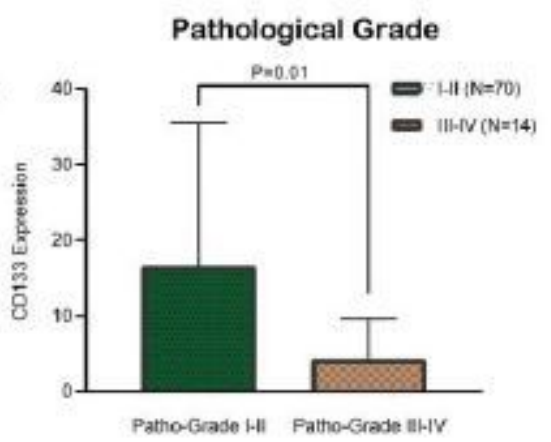

Tumor Length

G

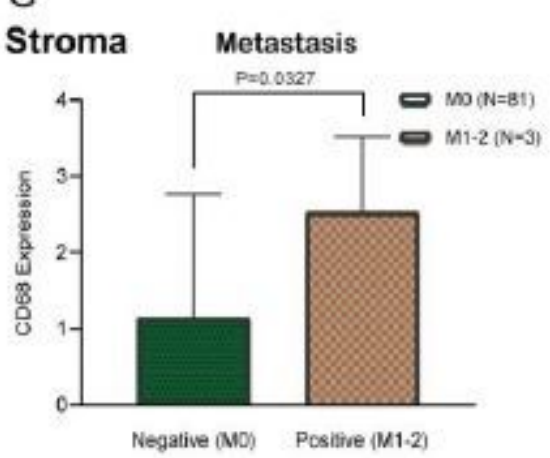

\section{Figure 6}

Significant correlations between four markers and clinicopathological characteristics in three cell classifications of CRC cancer tissues. A-D. In Total cells of cancer tissue; E-F. In Tumor cells of cancer tissue; G. In Stroma cells of cancer tissue. A and E. The expression of HER2 was increased in CRC patients at late T4 stage than those at early stages as T1, T2 and T3 . B. The expression of PD-L1 was decreased in small tumor with maximum Length $\geq 5 \mathrm{~cm}$ compared in large tumor $L<5 \mathrm{~cm}$ The levels of $C$. CD133 in Total similar to F. CD133 in Tumor, were reversely expressed in advanced pathological grading III, IV compared with in Grade I, II to D. CD68 in Total cells. G.CD68 expression was increased in CRC patients with distant metastasis than negative patients. 
A

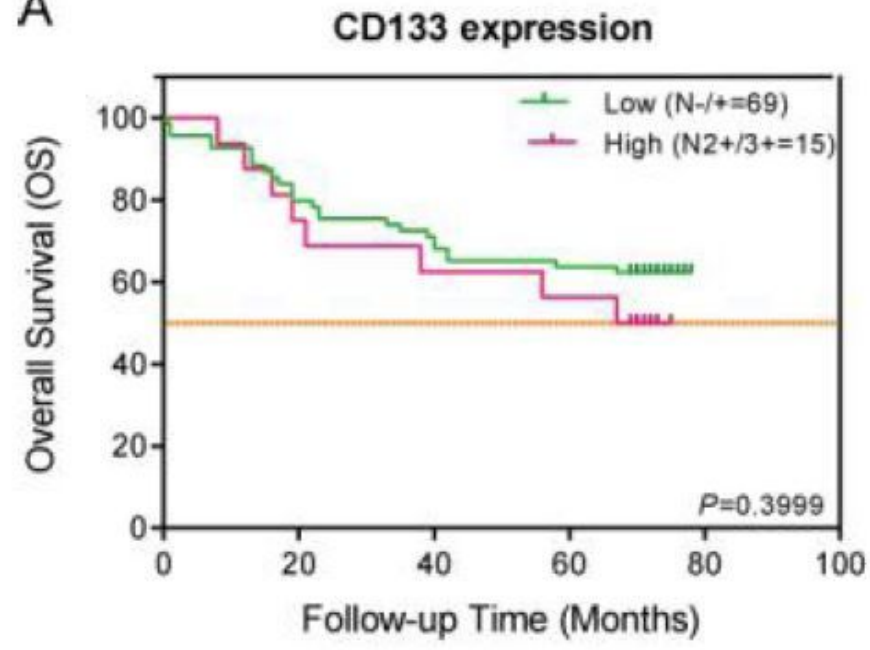

C

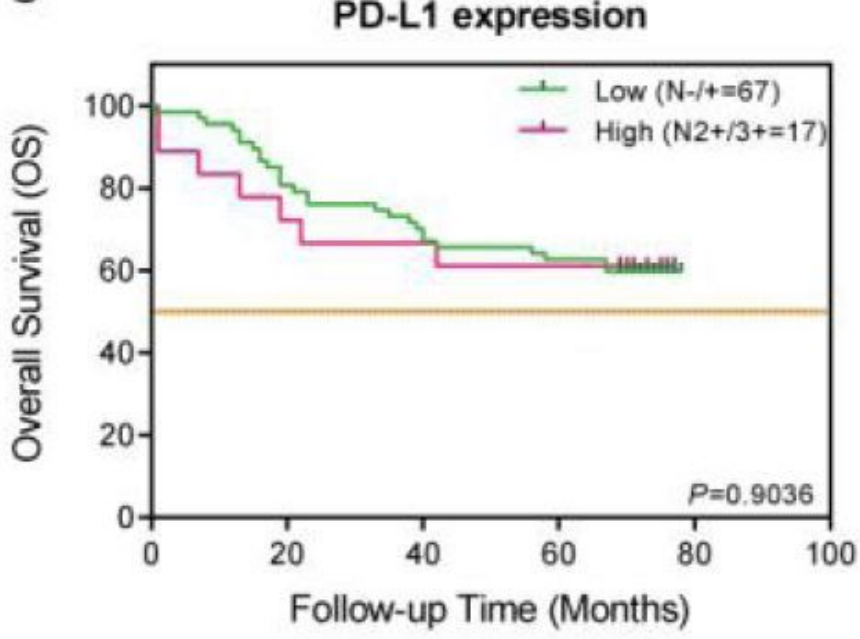

B

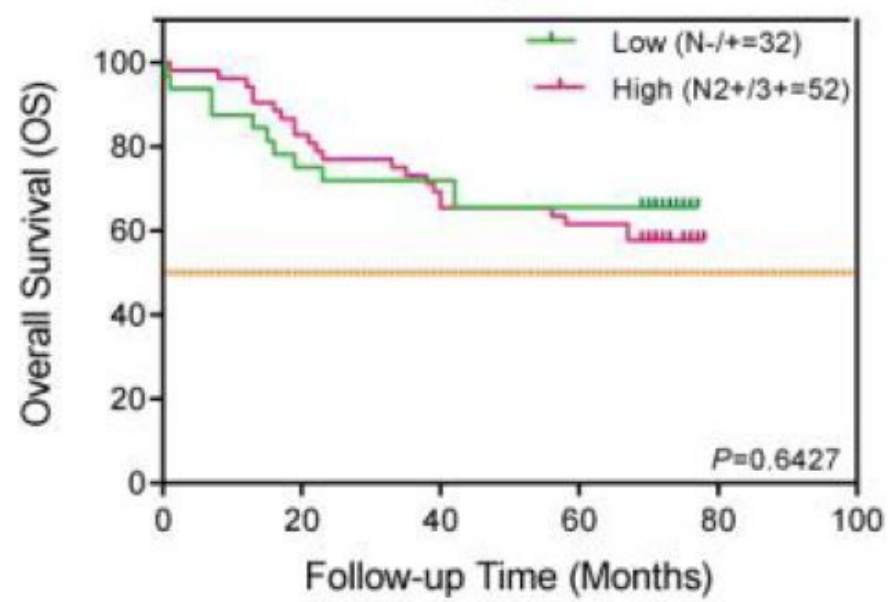

D

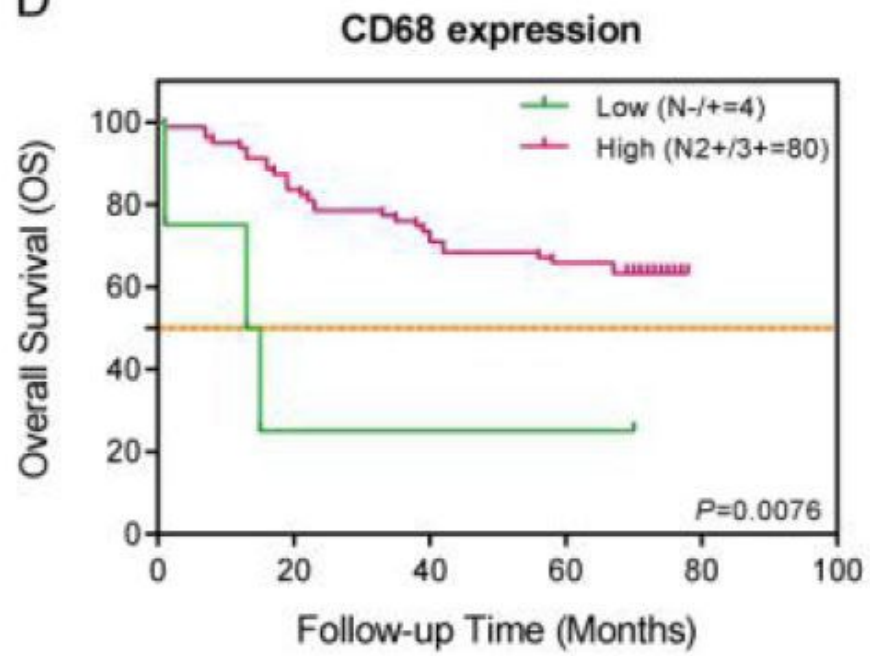

\section{Figure 7}

Kaplan-Meier analyzed OS rates with four marker expressions in CRC patients. The High- and Lowexpressions of A. CD133, B. HER2, C. PD-L1 and D. CD68, were associated with seven-year survival status in Total cells of Cancer tissues in 84 CRC patients. Orange dotted line: half of OS rates as $50 \%$. 